\title{
Emittance measurement and modeling for the Fermilab Booster
}

\author{
Xiaobiao Huang, ${ }^{1,2}$ S. Y. Lee, ${ }^{1,3}$ K. Y. Ng, ${ }^{2}$ and Y. $\mathrm{Su}^{1}$ \\ ${ }^{1}$ Department of Physics, Indiana University, Bloomington, Indiana 47405, USA \\ ${ }^{2}$ Fermi National Accelerator Laboratory, Box 500, Batavia, Illinois 60510, USA \\ ${ }^{3}$ GSI, Darmstadt, D64291, Germany
}

(Received 4 December 2005; published 30 January 2006)

\begin{abstract}
Turn-by-turn beam profile data measured at the Fermilab Booster are studied. Lattice models with experimental accelerator ramping parameters are used to obtain the lattice functions for data analysis. We studied the horizontal and vertical emittance growth behavior in different stages of a booster ramping cycle and its relation to the beam intensity. The transverse and longitudinal components in the horizontal beam width are separated by a fitting model which makes use of the different scaling rules of the beam momentum. We analyze the post-transition horizontal beam size oscillation based on a model where the longitudinal phase-space mismatch has resulted from rf voltage mismatch during the transition-energy crossing. We carried out systematic multiparticle simulation to show that the source of the vertical emittance growth is a combination of the random errors in skew-quadrupole and dipole fields, and the systematic Montague resonance. The effect of random quadrupole field is small for the Fermilab Booster because the betatron envelope tunes are reasonably far away from the half-integer stop band.
\end{abstract}

DOI: 10.1103/PhysRevSTAB.9.014202

PACS numbers: 52.59.Sa, 29.27. $-\mathrm{a}, 95.75 .-\mathrm{z}$

\section{INTRODUCTION}

Emittance evolution for space-charge-dominated beams is one of many important topics in high-intensity beam accelerators and storage rings (see Refs. [1-5]). Even a small percentage of beam loss in a high-intensity accelerator can cause radiation and operation problems. It is imperatively important to minimize beam loss and understand the emittance evolution.

There are many sources that can cause emittance growth. In linacs, the emittance growth can arise from synchrobetatron coupling, halo formation induced by collective envelope modes or structure resonances, etc. In circular accelerators, two of the most important candidates for the emittance growth are the half-integer stop band that can perturb the beam envelope function [1,2] and the Montague resonance [5]. There are many numericalsimulation codes that can be used to study the mechanism of emittance growth. However, systematic emittance evolution experiments are few in circular accelerators. Cousineau and collaborators have carried out systematic emittance measurement of high-intensity beams at the Proton Storage Ring (PSR) in Los Alamos National Laboratory and made systematic benchmarking of a space-charge simulation code [6]. Her conclusion indicated that the half-integer stop band played an important role in the emittance growth at the PSR.

There were no systematic emittance measurement for rapid cycling accelerators. The Fermilab Booster is a prototype of high power accelerator. It is a rapid cycling accelerator at $15 \mathrm{~Hz}$. Currently, the booster regularly delivers about $4 \times 10^{12}$ protons per pulse, with 10 -turn injection from a $400 \mathrm{MeV}$ linac. The overall efficiency is about $(83-85) \%$. At higher number of injection turns, the booster encounters substantial beam loss as shown in Fig. 1 mainly at the beginning of the acceleration cycle.

The injection efficiency and the efficiency of transitionenergy crossing derived from the measured total charge is shown in Fig. 2. Note that the efficiency of adiabatic capture is about $92 \%$ for injection turn less than 10, and decreases rapidly as the injection turn increases. Since the injection efficiency is nearly independent of the injection turn, the beam loss is most likely in the longitudinal phase space. Similarly, beam loss during the transition-energy crossing becomes more apparent when the injection turn is larger than 10. In the near future, the booster is required to

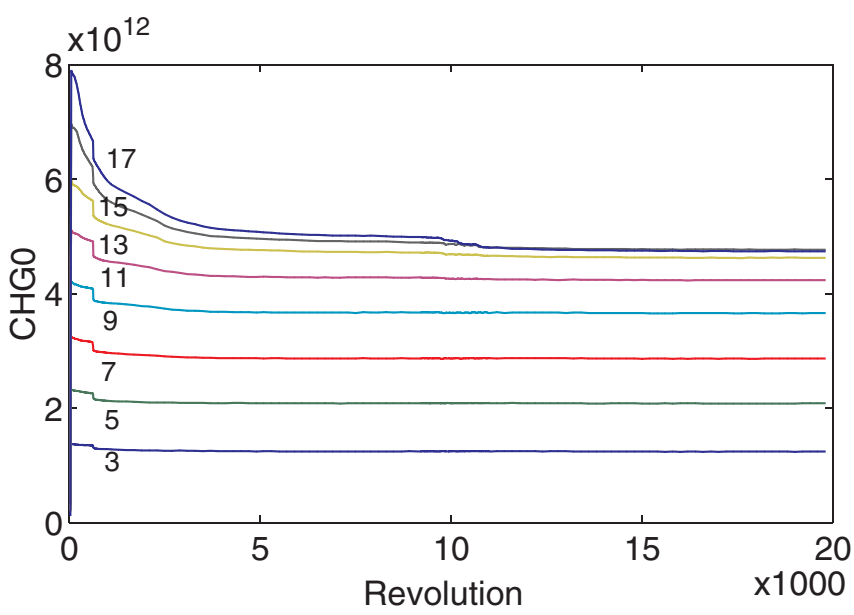

FIG. 1. (Color) Total charge (CHG0, in $10^{12}$ ) for data sets with $3,5,7$, etc., injection turns. Note that the notch of beam loss at about revolution-600 is created intentionally by kicking 3 bunches out to facilitate a gap for the extraction kicker. Transition-energy loss is visible at the transition energy at about 9550 revolution. 


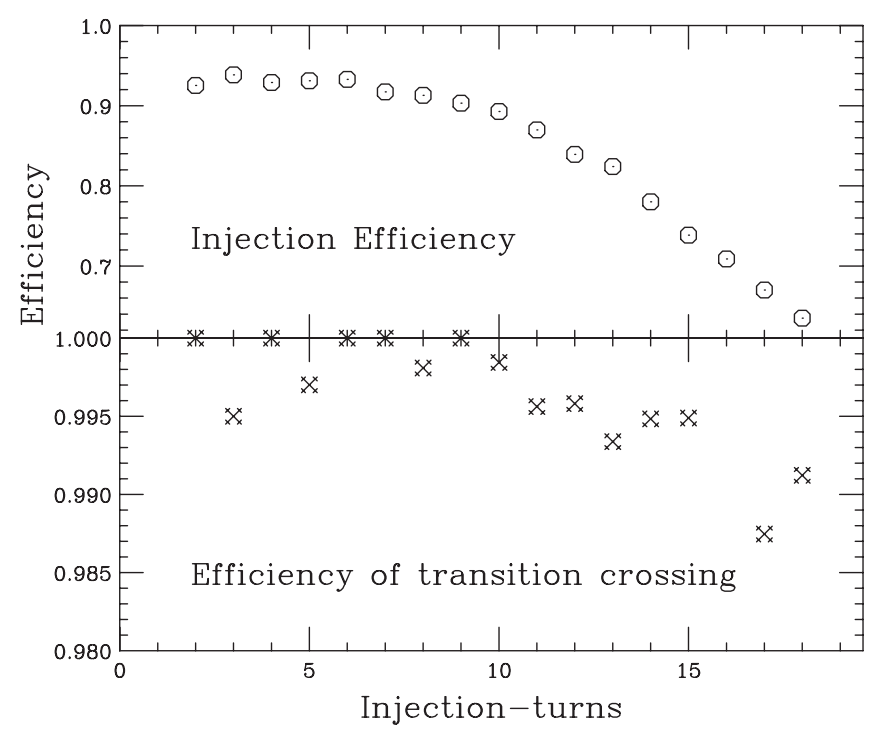

FIG. 2. The injection efficiency is defined as the ratio of the total charge at revolution 5000 divided by the total charge at injection excluding the 3 bunches intentionally kicked out. Note that the injection is nearly independent of the injection turn when the injection turn is less than 10 . The efficiency for transitionenergy crossing is defined as the ratio of the total charge at revolution 10010 and that at revolution 9010 .

deliver even higher proton flux for neutrino physics experiments.

This paper is intended to study the emittance evolution of the Fermilab Booster. The ionization profile monitor (IPM) is used to measure the horizontal and vertical beam size revolution by revolution. The measured $\mathrm{rms}$ beam profiles and widths are used to deduce the emittances of the beam. Using the deduced emittances, we will construct a model to fit these data and examine key ingredients which induce the emittance growth.

We organize this paper as follows. Section II presents beam profile measurement and method of emittance deduction. We build a realistic lattice model to analyze measured IPM data and deduce the horizontal and vertical emittances and the rms off-momentum width [7-9]. Section III discusses the algorithm and results of multiparticle tracking simulation. The space-charge force of our model is based on the potential derived from a Gaussian beam distribution, where the horizontal and vertical rms beam radii are updated in each revolution. We employ this particle tracking model to evaluate the effects of random errors in dipole, quadrupole, skew-quadrupole fields on the beam emittances. Section IV presents conclusions and discussions.

\section{THE EMITTANCE MEASUREMENTS}

IPM data for booster (under event 17 of the Fermilab Accelerator Control system) were taken with various intensity levels on 3 February 2005. The injection-turn num- bers were varied from 2 to 18 . The gate of the ionization profile monitor was about $1 \mu \mathrm{s}$, or the measured profile was the average of about 52 bunches. The experimental condition for all data sets was the same as the normal operational condition. The corrector package (e.g., trim quadrupole) settings were recorded to build a realistic lattice model for lattice function calculation.

The profile data at each revolution is fitted with a Gaussian plus polynomial model [10],

$$
p(y)=a+b y+A \exp \left(-\frac{\left(y-y_{0}\right)^{2}}{2 \sigma_{y}^{2}}\right),
$$

where $y$ is the transverse coordinate of a microstrip and $p$ is the reading of the strip. The separation of adjacent microstrips is $1.5 \mathrm{~mm}$ for the booster IPM. The parameters $\sigma_{y}$ and $y_{0}$ are half-rms width and central position of the beam, respectively. The calibration routine with linear parametrization is used to compensate the systematic error of beam width due to the space charge of the beam [10]. The calibration makes significant corrections for high intensity and small beam width cases. Thus the calibration is much more important for the horizontal beam profile, because $\beta_{x} \approx 6.5 \mathrm{~m} \ll \beta_{z} \approx 20.5 \mathrm{~m}$ and the dispersion $D_{x}=$ $1.8 \mathrm{~m}$ at the IPM location. Figure 3 shows typical calibrated beam profile for the 6-turn injection at revolutions 500, 4000, 8000, 12000 , and 16000, respectively.

The rms beam width $\sigma_{y}$ is related to the "emittance" by

$$
\epsilon_{y, \mathrm{rms}}=\frac{\sigma_{y}^{2}}{\beta_{y}}
$$

where $\epsilon_{y, \text { rms }}$ is rms emittance. The $95 \%$ emittance $\epsilon_{0.95}$ is $\epsilon_{0.95}=6 \epsilon_{\mathrm{rms}}$. These relations can be directly applied to vertical data $(y \rightarrow z)$. For horizontal data, the offmomentum width of the beam also contributes to the beam width. Assuming that the horizontal transverse phase-space distribution is uncorrelated to the longitudinal phase-space distribution, we obtain

$$
\left\langle x^{2}\right\rangle=\left\langle x_{\beta}^{2}\right\rangle+D^{2}\left\langle\delta^{2}\right\rangle, \quad \text { or } \quad \sigma_{x}^{2}=\beta_{x} \epsilon_{x, \mathrm{rms}}+D^{2} \sigma_{\delta}^{2},
$$

where $\langle\cdots\rangle$ denotes ensemble average, $\delta=\Delta p / p$ is the fractional momentum spread, $D$ is the value of the dispersion function at the IPM, and $x_{\beta}$ is the betatron part of the horizontal orbit, $\epsilon_{x, \text { rms }}$ is the rms horizontal emittance, and $\sigma_{\delta}$ is the rms fractional off-momentum width. The transverse emittances decrease as the beam momentum is ramped up due to adiabatic damping. The normalized emittance defined as $\epsilon_{\mathrm{n}}=\beta \gamma \epsilon$ is supposed to be conserved if nonconservative mechanisms are absent.

The charge signals were taken along with IPM data. Figure 1 shows the total charge for some data sets in the acceleration cycle. Note that loss occurs at the injection, and a small loss also occurs at high injection turn after 

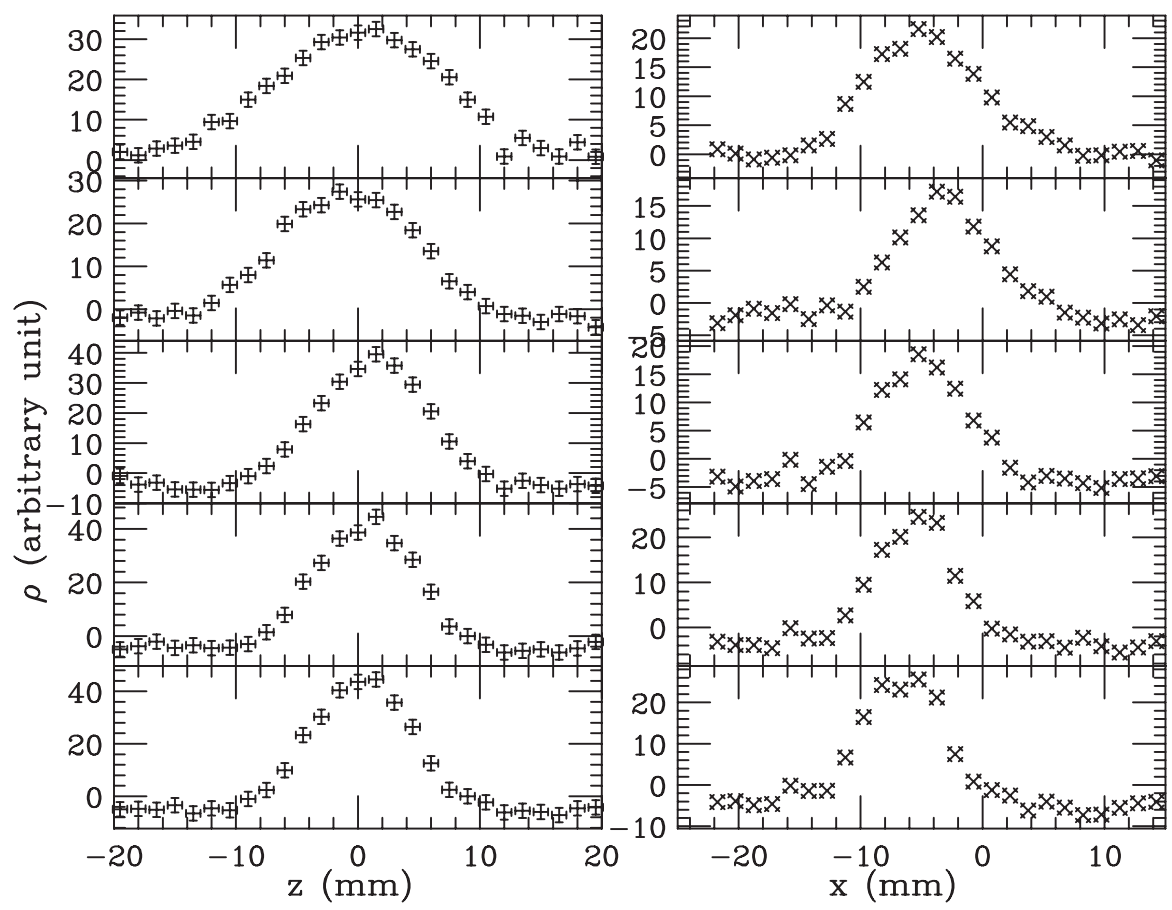

FIG. 3. The measured vertical and horizontal beam profiles for 6-turn injection measured at revolutions 500, 4000, 8000, 12 000, and 16000 are displayed from top to bottom, respectively.

transition crossing. Beam loss becomes very severe at injection turns larger than 12.

The transverse space-charge effect is characterized by the generalized space-charge perveance, defined as

$$
K_{s c}=2 N r_{0} /\left(\beta^{2} \gamma^{3}\right),
$$

where $N$ is number of protons per unit length and $r_{0}=$ $1.5347 \times 10^{-18} \mathrm{~m}$ is the classical radius of proton. Taking a Gaussian beam distribution with $N=N_{\mathrm{B}} /\left(\sqrt{2} \bar{\pi} \sigma_{s}\right)$,

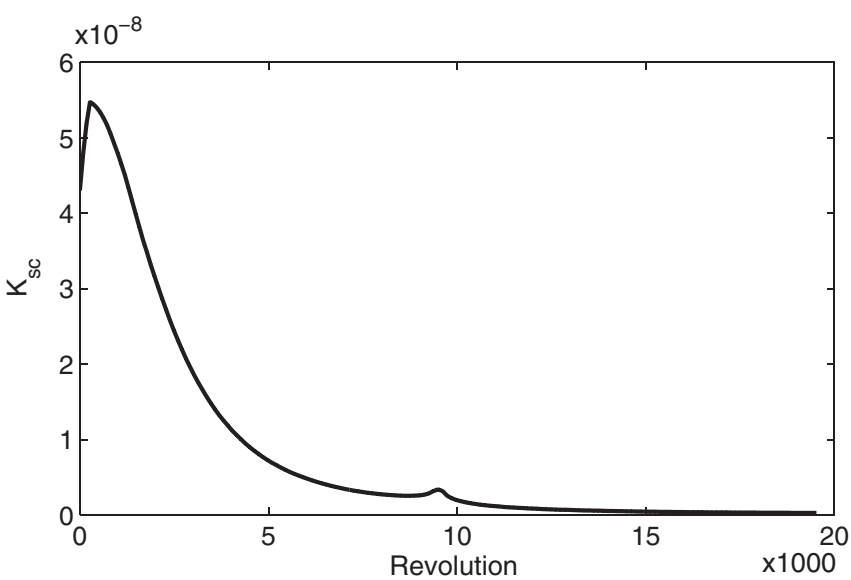

FIG. 4. The space-charge perveance parameter for an entire booster ramping cycle for the beam intensity of 10-turn injection (total charge of 4.1E12). The bunch length is derived from the total rf voltage (RFSUM) of the booster cycle while the $95 \%$ longitudinal emittance is assumed to be $0.08 \mathrm{eV}-\mathrm{s}$. where $N_{\mathrm{B}}$ is the number of particles in a bunch and $\sigma_{s}$ is the longitudinal rms bunch length, we plot the spacecharge perveance for a booster cycle for the beam intensity with 10-turn injection in Fig. 4.

For emittance calculation in the entire cycle, we build the lattice model at $1 \mathrm{~ms}$ interval in the cycle according to the actual beam energy and the accelerator lattice setting. The lattice functions such as beta-functions $\beta_{x}, \beta_{z}$, dispersion function $D$ and transition gamma $\gamma_{t}$ anywhere in the cycle are calculated with interpolation. Figure 5 shows

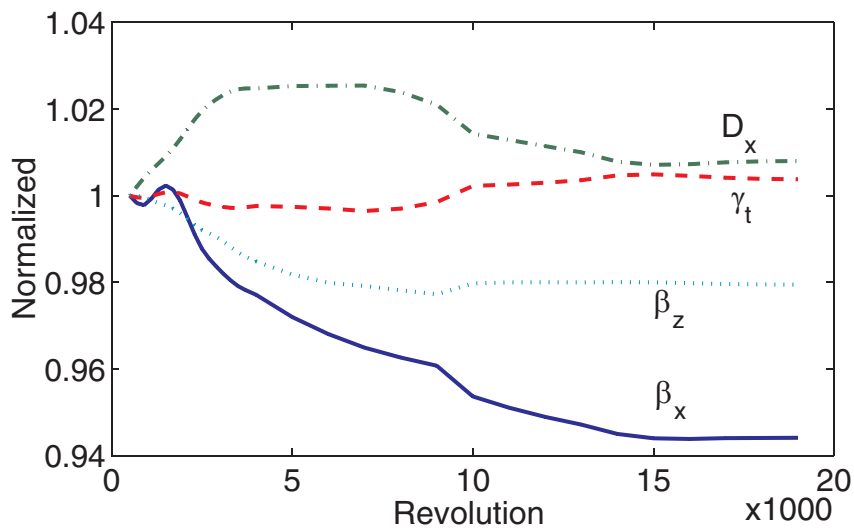

FIG. 5. (Color) Lattice functions $\beta_{x}, \beta_{z}, D_{x}$ at the IPM location and the transition $\gamma_{t}$ normalized by the values at the first $1 \mathrm{~ms}$, where $\beta_{x 0}=6.5 \mathrm{~m}, \beta_{z 0}=20.5 \mathrm{~m}, D_{x 0}=1.8 \mathrm{~m}$ and $\gamma_{t 0}=$ 5.48. The change of these lattice functions arises from the effect of the dc dogleg magnets (extraction chicane) and the trim quadrupoles. 


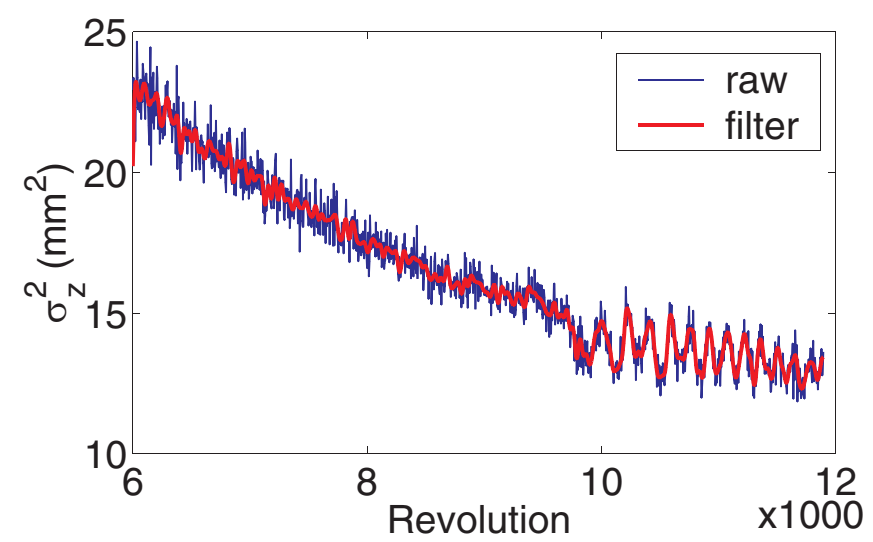

FIG. 6. (Color) The raw (blue) and filtered $\sigma_{z}^{2}$ (red) for 10-turn injection are shown for comparison.

the change of these lattice functions in the cycle at the IPM location.

The revolution-by-revolution beam-width signal from the IPM is usually noisy. Since we are interested only in the average behavior, it is useful to filter out the highfrequency noises. We apply a 40-points low-pass digital filter with tune threshold 0.1 to remove such noises. Figure 6 shows the effects of the filter for the vertical mean-square beam size for 10-turn injection.

\section{A. The vertical emittance}

Since the transverse emittances are adiabatically damped as the beam momentum increases, it is more convenient to study the normalized emittance. Figure 7 shows the normalized vertical rms emittance for two data sets with 4-turn or 12-turn injection, respectively. We note the emittance starts with the same level $(2 \pi \mathrm{mm} \mathrm{mrad})$ but follows different growth pattern for different intensities.

The normalized emittance grows rapidly in the first 4000 revolutions because of the space-charge effect and the growth rate is nonlinear and highly dependent on beam

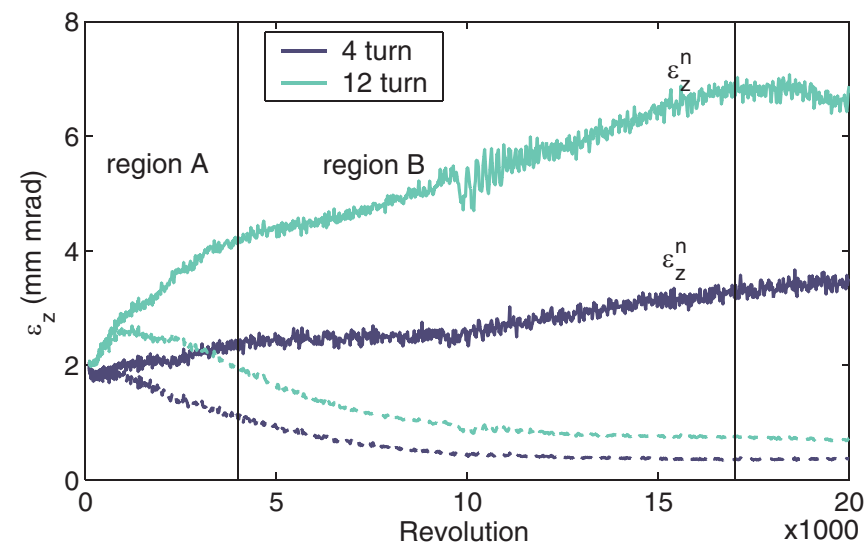

FIG. 7. (Color) The vertical rms emittance (dashed) and the normalized rms emittance (solid) in the entire cycle for 4-turn and 12-turn injection. intensities. For the later part of the cycle (4001-17000 revolutions), except close to extraction, the growth is slow and linear. The cause of the later emittance growth is not likely the space-charge effect because the relativistic gamma is large enough in this region. Possible growth mechanisms are residual gas scattering, intrabeam scattering, or other mechanisms. We will treat the two growth periods separately with the first period (region A) from 70 revolution after injection to 4000 revolution and the second period (region B) from 4001 revolution to 17000 revolution.

\section{Linear growth region}

The emittance growth in region B can be roughly considered as linear. However some data sets may lose beam during the transition-energy crossing and some have posttransition oscillation. We fit the pretransition and posttransition regions to linear curves separately. The fitting curves are shown in Fig. 8.

The growth rate of emittance defined by

$$
\alpha_{z}=\frac{\Delta \epsilon_{z}^{n}}{\Delta N}
$$

where $N$ is the revolution number, is calculated with the slopes of the linear curves. The growth rates are shown in Fig. 9. The vertical normalized emittance grows about $1 \pi \mathrm{mm} \mathrm{mrad}$ in $10^{4}$ revolutions for normal working cycles of the booster. The growth rate seems to be proportional to the injection turns.

The growth rate due to residual gas scattering is estimated to be $\Delta \epsilon^{n} /\left(\epsilon^{n} \Delta t\right)=0.008 \mathrm{~s}^{-1}$ at the vacuum pressure of $100 \mathrm{nTorr}$, which is about $1.2 \times 10^{-4}$ in $15 \mathrm{~ms}$ (or 10000 revolutions). Thus the emittance growth can not be caused by the residual gas small-angle scattering. The growth rate due to the intrabeam scattering is estimated to be less than $\Delta \epsilon^{n} / \epsilon^{n} \Delta t \leq 0.001 \mathrm{~s}^{-1}$ [11]. It is also difficult to see the growth rate of the emittance as large as $1 \pi \mathrm{mm}$ mrad in $10^{4}$ revolutions.

\section{Space-charge-dominated region}

Space-charge effects play an important role in the first 4000 revolutions. Figure 10 shows the normalized vertical rms emittance in region A for all data sets. Note that with 12-turn injection or less, the emittance growth is relatively mild. The growth behavior for these data sets follows a similar pattern, which could be modeled. However, for intensity higher than 13-turn injection, the emittances grew and beam loss occurred in the first few hundreds of revolutions as shown in Fig. 1.

Since the 1960s, emittance dilution due to space charge has been a subject of intensive studies, the exact emittance growth mechanism is not clear, and may even be different in each accelerator. A popular belief is that the emittance dilution has resulted from the half-integer stop band of the betatron envelope function [1,2]. The stop band width 

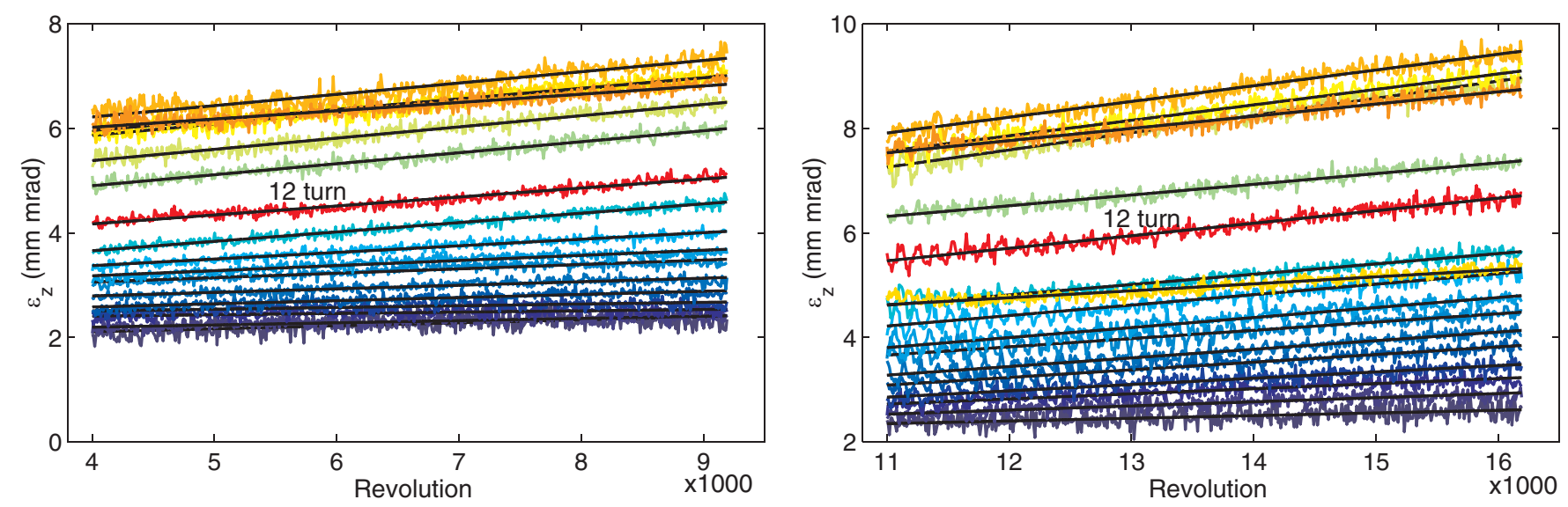

FIG. 8. (Color) Left: The normalized vertical rms emittance from 4001 revolution to 9200 revolution for all data sets with 2-turn injection to 18-turn injection. Right: The normalized vertical rms emittance from 11001 revolution to 16200 revolution for all data sets with 2-turn injection to 18-turn injection. Note 16-turn data set lost a big fraction of beam at transition and thus has smaller emittance here.

depends on the distribution of quadrupole field in an accelerator, while the betatron envelope-tune spread is proportional to the space-charge perveance $K_{\mathrm{sc}}$, which depends on the bunching factor and the beam momentum.

In this section, we will use a phenomenological approach to extract properties of emittance growth by assuming that the instantaneous emittance growth rate is proportional to $K_{\mathrm{sc}}$ :

$$
\frac{d \epsilon}{d t}=b_{1}+b_{2} K_{\mathrm{sc}}
$$

where $t$ is the revolution number. The normalized emittance can then be expressed as

$$
\epsilon_{z}=a_{0}+b_{1} t+b_{2} \int_{0}^{t} K_{\mathrm{sc}} d t^{\prime}
$$

where $a_{0}$ denotes initial normalized emittance, $b_{1}$ denotes linear growth rate and $b_{2}$ denotes space-charge-induced

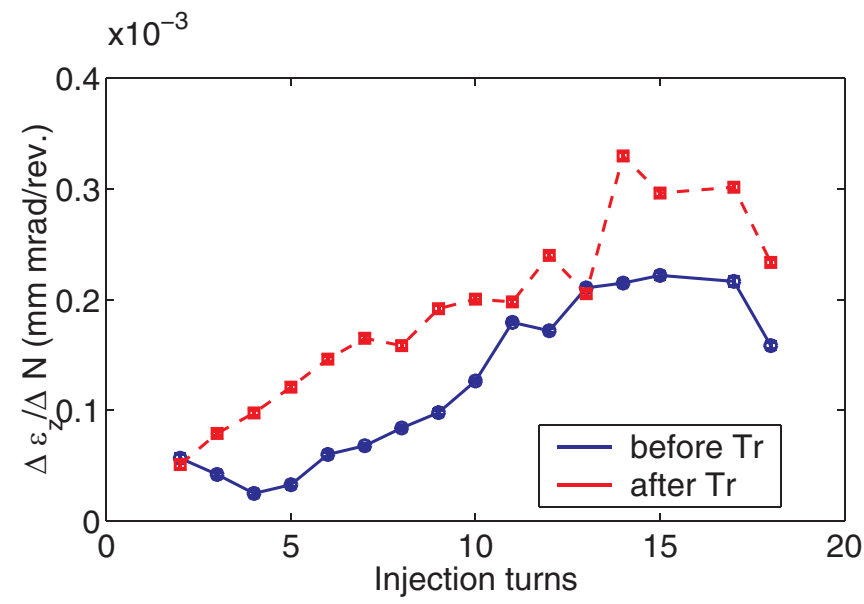

FIG. 9. (Color) The vertical emittance growth rate before (blue) and after (red) transition for all data sets. growth rate. We fit the measured $\epsilon_{z}^{n}$ to this model from 2turn up to 11-turn injections. Beyond 11-turn injection, beam loss at injection becomes substantial. A typical fitting curve is shown in Fig. 11.

The fitting parameters are shown in Fig. 12. Note that the initial emittance varies from $1.6 \pi \mathrm{mm} \mathrm{mrad}$ to $2.0 \pi \mathrm{mm}$ mrad for all data set. The fitted linear growth rate agrees well with that obtained in the linear growth region, shown in Fig. 9. The space-charge-dependent growth rate $b_{2}$ is nearly constant for all data sets because the intensity has been absorbed by the perveance parameter $K_{s c}$.

An alternative model, based on Eq. (6.16) of Ref. [12], leads to

$$
\left(\epsilon_{z}^{n}\right)^{2}=\tilde{a}_{0}\left(1+\tilde{b}_{1} t+\tilde{b}_{2} \int_{0}^{t} K_{s c} d t^{\prime}\right)
$$

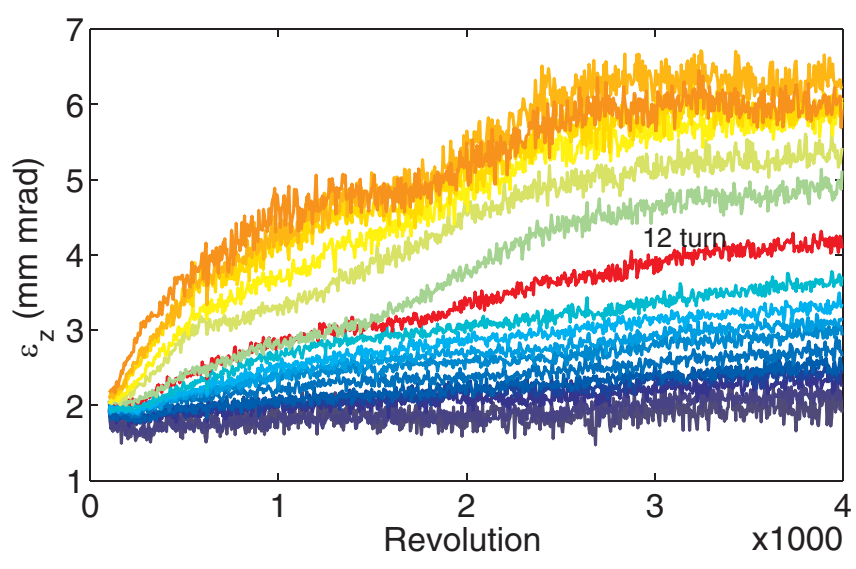

FIG. 10. (Color) The normalized vertical rms emittance from 70 revolution to 4000 revolution for all data sets with 2-turn injection to 18-turn injection. Note the red curve is for 12-turn injection which marks the border of two kinds of emittance growth behavior. 


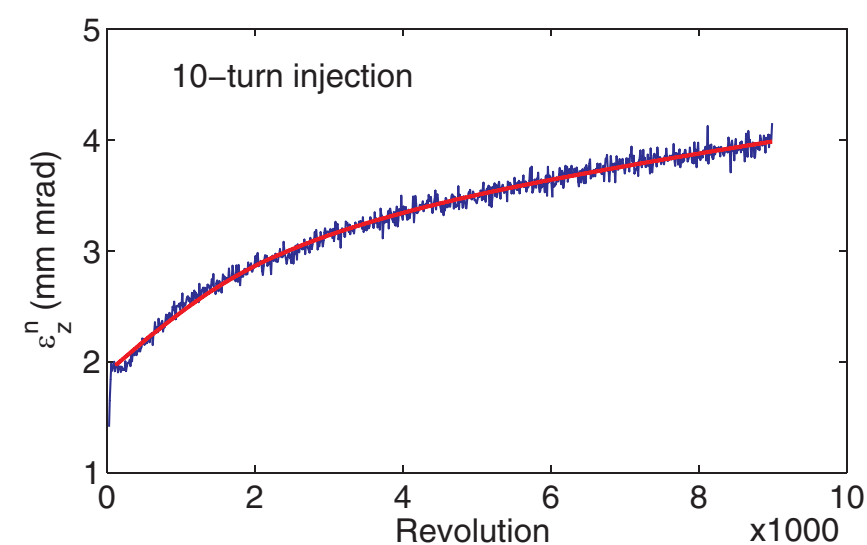

FIG. 11. (Color) A typical fitting curve of the model using Eq. (6) for the data set with 10-turn injection.

can also be used to fit the data well. This model describes emittance growth due to an initial nonequilibrium distribution of the beam. Since both $\tilde{b}_{1}$ and $\tilde{b}_{2}$ parameters are small, the resulting fits are equivalent to the model of Eq. (6).

\section{B. Horizontal emittance}

The horizontal beam width reflects both the horizontal emittance and the longitudinal off-momentum distribution as seen in Eq. (3). Figure 13 shows $\sigma_{x}^{2}$ for 4-turn and 12turn injection for the entire ramping cycle. The offmomentum width becomes more important around transition where the bunch length is shortened. The beam width

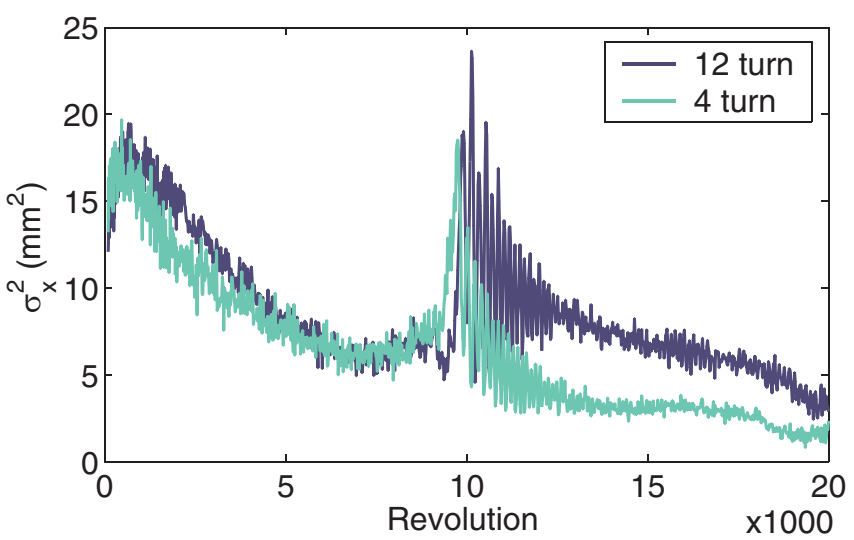

FIG. 13. (Color) $\sigma_{x}^{2}$ for 4-turn and 12-turn injection. The beam widths below and above transition energy shows adiabatic damping, while the beam width is enhanced at the transition-energy region due to the increase of the momentum width.

starts oscillating after transition because of the longitudinal phase-space mismatch.

Since the horizontal beam width is a quadrature of contributions from the betatron motion and the rms offmomentum oscillation, we cannot directly calculate the normalized horizontal emittance unless we can isolate and remove the contributions of the off-momentum contribution. In the following, we will discuss a method by taking the advantage of different scaling property as a function of the beam momentum. We will show that the momentum scaling property can effectively separate the
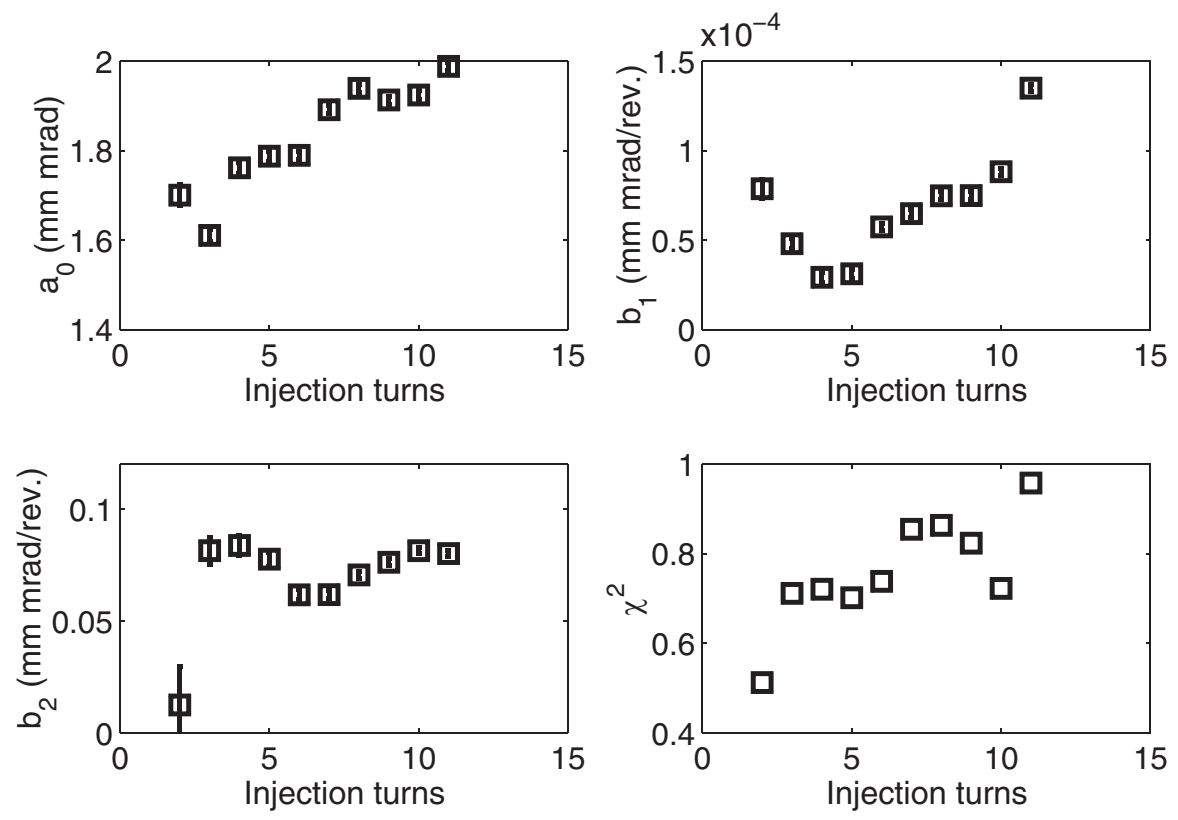

FIG. 12. Top left: Parameter $a_{0}$, initial vertical normalized emittance. Top right: parameter $b_{1}$, linear growth rate. Bottom left: Parameter $b_{2}$, space-charge-dependent growth rate. Bottom right: the residual $\chi^{2}$, normalized by noise sigma and number of data points. 


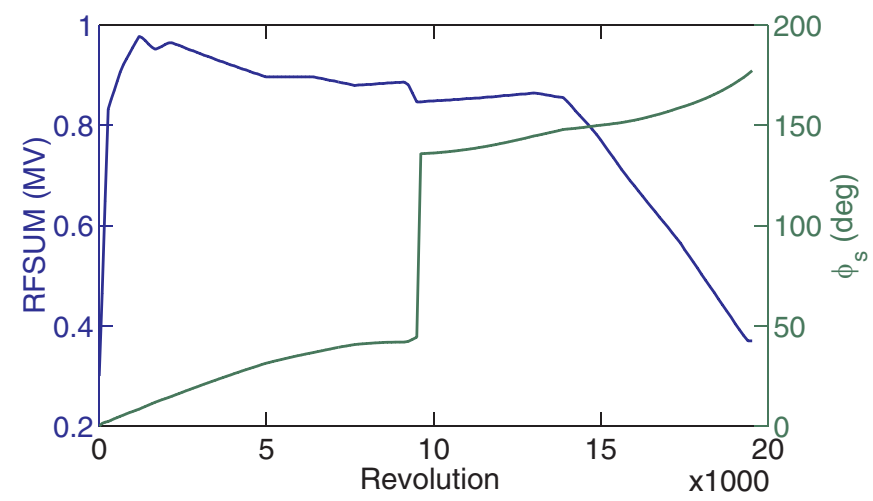

FIG. 14. (Color) The rf voltage $V$ (RFSUM) and the synchronous phase $\phi_{s}$ recorded with console program during this experiment.

transverse horizontal betatron emittance and the offmomentum width.

\section{Below transition energy}

One way to obtain the transverse betatron component in $\sigma_{x}^{2}$ is to subtract the "known" off-momentum width component, i.e., $\sigma_{x, \beta}^{2}=\sigma_{x}^{2}-D^{2} \sigma_{\delta}^{2}$. We can do so by assuming the beam fills up the rf bucket during the adiabatic capture at injection. The bunch shape then follows the evolution of rf bucket as a matched beam, which can be determined by the known rf voltage $V$ and rf synchronous phase $\phi_{s}$. Figure 14 shows the rf voltage (RFSUM), recorded with console program during the experiment, and the rf synchronous phase, calculated by the energy gain per revolution according to the magnetic field ramp. The top plot of Fig. 15 shows the rf bucket area. The corresponding rms off-momentum width and bunch length (assuming that the $95 \%$ bunch phase-space area of $0.08 \mathrm{eV}-\mathrm{s}$ ) is shown in the bottom plot of Fig. 15. The longitudinal component in $\sigma_{x}^{2}$ can then be calculated with $D^{2} \sigma_{\delta}^{2}$.

Another way is to make use of the difference of the scaling rules of the transverse and longitudinal components [2]. Both terms of Eq. (3) change in the cycle as the momentum is ramped up. However, they change in different scaling rules:

$$
\sigma_{x}^{2}=\beta_{x} \epsilon_{\mathrm{rms}}+D^{2} \sigma_{\delta}^{2}=a A(t)+b B(t)
$$

with

$$
\begin{gathered}
a=\epsilon_{\mathrm{rms}}^{n} \frac{\beta_{x 0}}{\beta_{0} \gamma_{0}}, \quad A(t)=\frac{\beta_{x}}{\beta_{x 0}} \frac{\beta_{0} \gamma_{0}}{\beta \gamma}, \\
b=D^{2} \sigma_{\delta 0}^{2}, \quad B(t)=\frac{\gamma_{0} \sqrt{\gamma_{0}\left|\eta_{0}\right| / V_{0}\left|\cos \phi_{s 0}\right|}}{\gamma \sqrt{\gamma|\eta| / V \mid \cos \phi_{s}}},
\end{gathered}
$$

where we have used the scaling rule of $\sigma_{\delta} \sim$ $V^{1 / 4}|\eta|^{-1 / 4} \gamma^{-3 / 4}$ [2]. The scaling functions $A(t)$ and $B(t)$ are shown in Fig. 16.

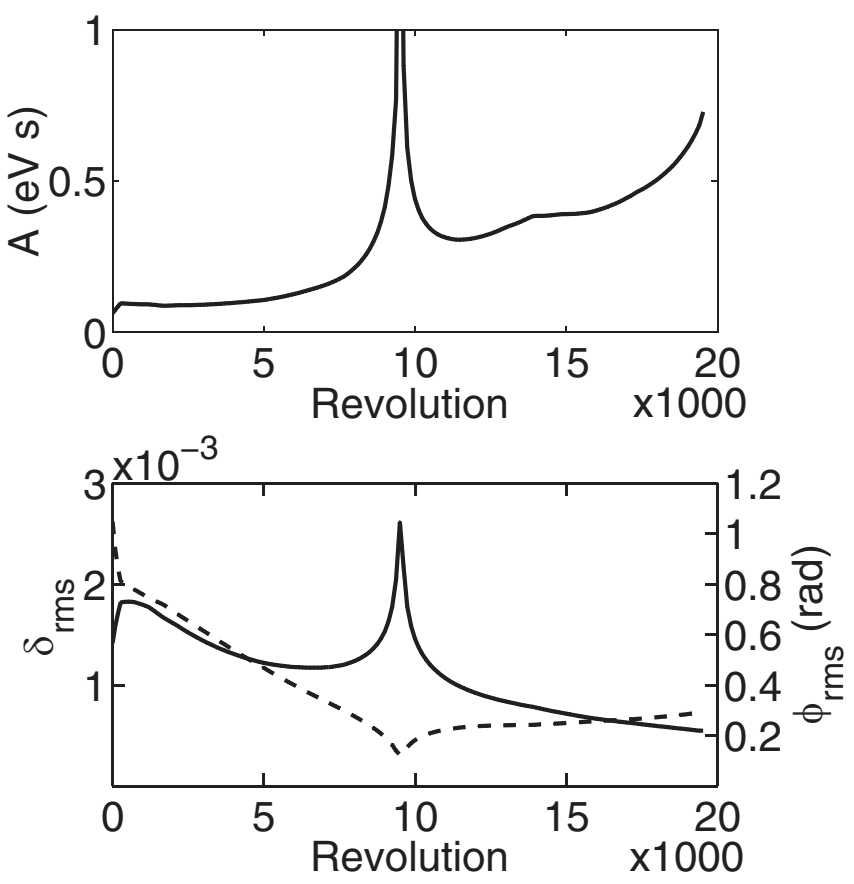

FIG. 15. Top: The bucket area throughout the booster cycle. The bucket area is about $0.08 \mathrm{eV}$-s at the beginning of the ramping cycle. Bottom: The rms momentum width $\sigma_{\delta}$ (solid) and rms bunch length $\sigma_{\phi}$ (dashed), assuming phase-space area $A=0.08 \mathrm{eV}$-s. The rms value is obtained by dividing the maximum $\delta$ and $\phi$ by $\sqrt{6}$.

The normalized horizontal emittance can be considered as growing linearly if it has the same behavior as the vertical emittance, so we further assume $a=a_{0}+a_{1} t$. The invariant longitudinal phase-space area is preserved during acceleration, i.e., $b=b_{0}$. This assumption is reflected in the fact that the bucket area is nearly constant during the first 8000 revolutions in the booster acceleration cycle. If there is any longitudinal phase-space dilution, these particles will be squeezed out of the bucket. We thus try to fit the horizontal width with

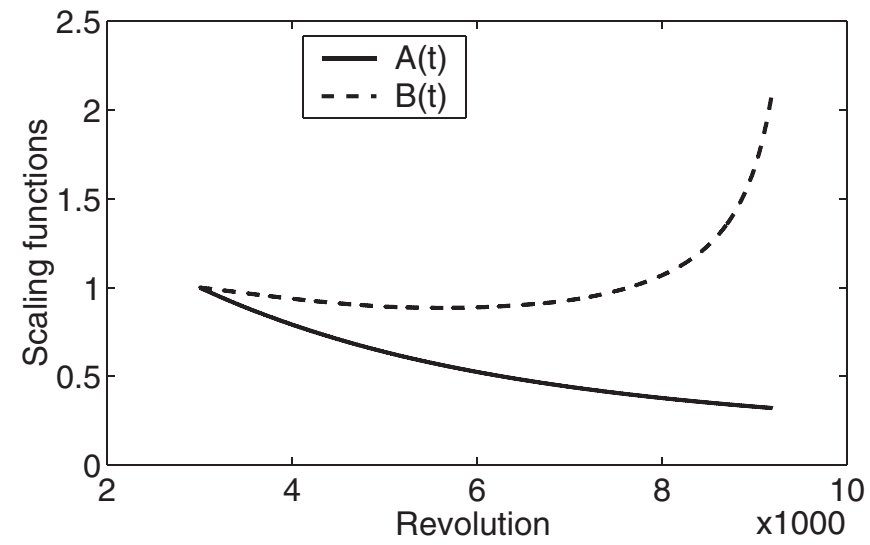

FIG. 16. Scaling function $A(t)$ and $B(t)$, obtained with realistic booster lattice model and rf parameters. 


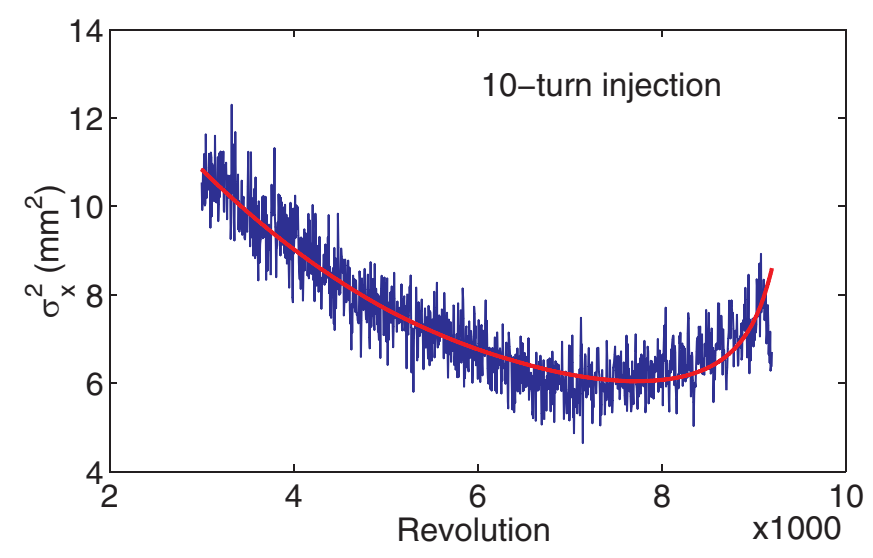

FIG. 17. (Color) An example of the fitting curves using Eq. (11) for the horizontal data sets.

$$
\sigma_{x}^{2}=\left(a_{0}+a_{1} t\right) A(t)+b_{0} B(t)
$$

To avoid the nonlinear emittance blowup in the first several milliseconds of the cycle and the nonadiabatic region near transition, we fit $\sigma_{x}^{2}$ from 3001 revolution to 9200 revolution to the model of Eq. (11) to obtain constant parameters $a_{0}, a_{1}$, and $b_{0}$ for each data set. We then convert these parameters to horizontal emittance or rms momentum width according to Eqs. (9) and (10). An example of fitting curves is shown in Fig. 17.

This model does not fit the data of high-intensity data very well because the calibrated result of the linear parametrization scheme may deviate from the actual beam size for high-intensity beams (see Fig. 2 in Ref. [10]). The horizontal beam size $\sigma_{x}$ (see Fig. 13) drops from $3.5 \mathrm{~mm}$ (at 3001 revolution) to $2.5 \mathrm{~mm}$ at around 7000 revolution. For a beam with $\sigma_{x}=3 \mathrm{~mm}$ at a total charge of $4 \times 10^{12}$ particles (10-turn injection), the calibration error is as large as $5 \%$. As the beam size shrinks due to adiabatic damping, the calibration error of IPM data gets even worse. The scaling property is particularly sensitive to errors in the IPM data calibration. Thus we fit data sets with 10-turn injection or less. The resulting normalized emittance, its growth rate and the rms momentum width are shown in Fig. 18. The standard deviations of the noises in $\sigma_{x}^{2}$, along with the covariance matrix of the fitting, are used to estimate the error bars of these parameters.

The results give reasonable values of emittances and rms momentum widths at 3001 revolution. The horizontal normalized emittances are found to be about $2 \pi \mathrm{mm}$ mrad. It seems that the explosive emittance blowup observed in the vertical plane (see Sec. II A), due to the space-charge effects, is absent in the horizontal data. This horizontal emittance at 3001-revolution number is about the same as that of the initial vertical emittance (see the top-left plot of Fig. 12). The emittance growth rate, defined as

$$
\alpha_{x}=\frac{\Delta \epsilon_{x}^{n}}{\Delta N},
$$

is about $0.8 \pi \mathrm{mm}$ mrad per $10^{4}$ revolutions. The horizontal growth rate is about the same as the vertical growth rate shown in Fig. 9. The rms momentum width is about $1.0 \times$ $10^{-3}$, which is smaller than the value $1.4 \times 10^{-3}$ as predicted in Fig. 15. The momentum width for these data sets
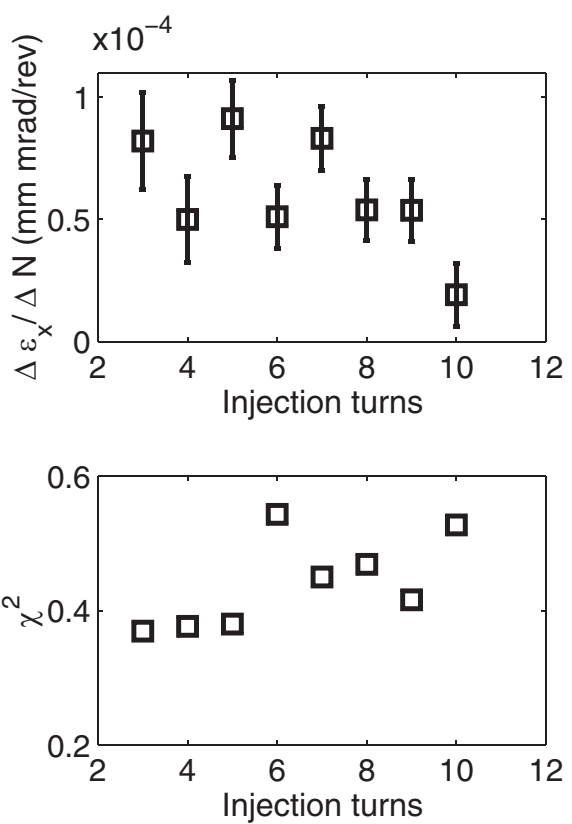

FIG. 18. Top left: the fitted normalized horizontal emittance at 3001 revolution $\epsilon_{0}=a_{0}\left(\beta_{0} \gamma_{0} / \beta_{x 0}\right)$. Top right: the horizontal emittance growth rate $\Delta \epsilon_{x}^{n} / \Delta N$. Bottom left: the fitted rms momentum width $\sigma_{\delta}=\sqrt{b_{0}} / D_{0}$ at the 3001 revolution. Bottom right: the normalized residual $\chi^{2}$ for all cases. 
are nearly equal, indicating the rf buckets are nearly filled with particles at injection.

We have measured the momentum spread using the resistive wall monitor signal with a high resolution scope for 4-turn and 11-turn injection. The recorded peaks on the beam current signal are fitted to elliptic model [13] to derive the bunch lengths. The bunch lengths become the momentum spread according to the phase-space ellipses. The results are shown in Fig. 19. The rms momentum spread at 3001 revolution, deduced from the wall monitor data, is $1.25 \times 10^{-3}$.

From the above analysis, we find that the scaling law can successfully be used to separate the horizontal betatron and off-momentum components in the horizontal beam width. The essential error arises from the fact the IPM is located at a small $\beta_{x}$ location, and thus it is intrinsically more prone to errors. The resulting horizontal emittance and rms offmomentum width deduced from the IPM data agree well with other independent measurements.

\section{Across the transition energy}

The rms momentum width starts to grow rapidly as the beam gets near transition, where the phase slip factor $\eta$ becomes small. The bunch shape cannot follow the rf bucket when it is very close to transition and the longitudinal motion is nonadiabatic. The adiabatic time and nonlinear time for booster are estimated to be [2]
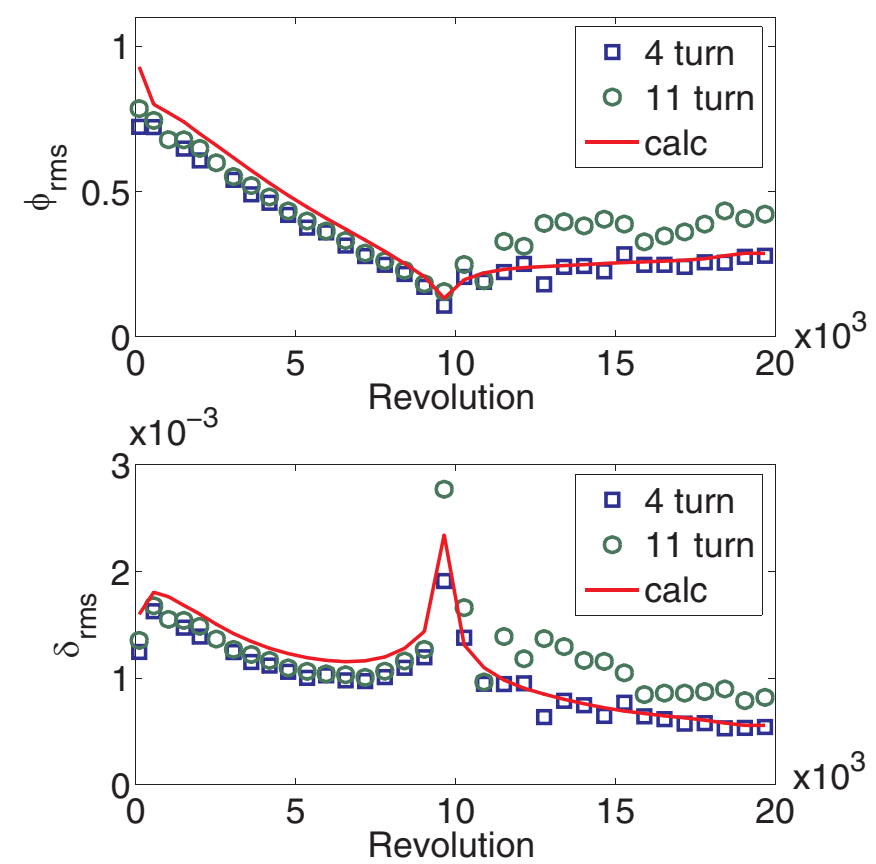

FIG. 19. (Color) The measured rms bunch length $\sigma_{\phi}$ (top) and rms momentum width $\sigma_{\delta}$ (bottom) for 4-turn and 11-turn injection are compared to those calculated with phase-space area $0.08 \mathrm{eV}$-s. The measurements were taken on 29 April 2005 under event 14.

$$
\begin{aligned}
\tau_{\mathrm{ad}} & =\left(\frac{\pi \beta^{2} m c^{2} \gamma_{\mathrm{T}}^{4}}{\dot{\gamma} \omega_{0}^{2} h e V\left|\cos \phi_{\mathrm{s}}\right|}\right)^{1 / 3} \approx 0.187 \mathrm{~ms}, \\
\tau_{\mathrm{nl}} & =\gamma_{\mathrm{T}}^{3} \frac{\eta_{1}}{2 \dot{\gamma}} \hat{\delta}=\gamma_{\mathrm{T}} \frac{\frac{3}{2} \beta_{0}^{2}+\gamma_{\mathrm{T}}^{2} \alpha_{1}}{2 \dot{\gamma}} \hat{\delta} \approx 0.100 \mathrm{~ms},
\end{aligned}
$$

where we use $\dot{\gamma} \approx 437 \mathrm{~s}^{-1}, \gamma_{\mathrm{T}}^{2} \alpha_{1}=1.0$, and $\hat{\delta}=\sqrt{6} \sigma_{\delta}$. The maximum rms momentum width at transition is obtained by using the nonadiabatic formula

$$
\begin{aligned}
\left.\sigma_{\delta}\right|_{\gamma=\gamma_{\mathrm{T}}} & =\frac{\gamma_{\mathrm{T}}}{3^{1 / 6} \beta \tau_{\mathrm{ad}} \Gamma\left(\frac{2}{3}\right)}\left(\frac{2 \mathcal{A}}{3 m c^{2} \dot{\gamma}}\right)^{1 / 2} \\
& \approx 0.502 \frac{\gamma_{\mathrm{T}}}{\beta \tau_{\mathrm{ad}}}\left(\frac{\mathcal{A}}{m c^{2} \dot{\gamma}}\right)^{1 / 2} .
\end{aligned}
$$

Here $\mathcal{A}$ is the rms phase-space area of the beam in $\mathrm{eV}$-s. We obtain $\tau_{\mathrm{ad}}=0.187 \mathrm{~ms}$, and $\tau_{\mathrm{nl}}=0.100 \mathrm{~ms}$, and the total growth due to nonlinear longitudinal motion is $G \approx$ $\exp \left\{\frac{2}{3}\left(\tau_{\mathrm{nl}} / \tau_{\mathrm{ad}}\right)^{3 / 2}\right\} \approx 1.30 \quad$ [2]. Thus the phase-space growth due to the nonlinear motion is small. Figure 19 shows clearly that the measured rms bunch length and offmomentum spread agree very well with those derived with a constant phase-space area for the 4-turn injection case.

\section{Microwave instability}

The beam near transition energy can also suffer microwave instability. The emittance growth factor can be estimated as $G=\exp (S)$, where $[14,15]$

$$
S \approx \frac{n \omega_{0} N_{\mathrm{B}}^{2} e^{4}\left|Z_{\|} / n\right|^{2} \gamma_{\mathrm{T}}^{3}}{16 \pi \sigma_{\delta 0} \mathcal{A}^{2} \dot{\gamma}} F,
$$

where $\mathcal{A}=\pi \sigma_{E} \sigma_{t}$ is the rms phase-space area in (eV-s), $F=0.207$ is the form factor, $N_{\mathrm{B}}$ is the number of particles in a bunch, and $n=R / b \approx 1500$ is the mode number. Assuming a broadband impedance of $\left|Z_{\|}\right| / n \approx 20 \Omega$, $6 \mathcal{A}=0.08 \mathrm{eV}$-s, $\sigma_{\delta 0} \approx 0.00232$ is the rms momentum spread at the longitudinal center of the bunch, and $N_{\mathrm{B}}=$ $6 \times 10^{10}$, we find $G \approx 2.2$, which is small because the microwave instability growth is the growth factor on the very small Schottky noise.

\section{Bunch mismatch oscillations in the synchrotron phase space}

The post-transition beam width oscillations may arise from the mismatch across transition due to longitudinal space-charge potential as pointed out by Sorensen [16]. In a linearized approximation, the longitudinal Hamiltonian around the transition-energy region is

$$
\begin{aligned}
H(\phi, \delta) \approx & \frac{h \eta \omega_{0}}{2} \delta^{2}-\frac{\omega_{0} e}{4 \pi \beta^{2} E}\left[V \cos \phi_{\mathrm{s}}-\frac{h e c g_{0} Z_{0} N_{\mathrm{B}}}{2 \gamma^{2} R \sigma_{\phi}^{3}}\right] \\
& \times\left(\phi-\phi_{s}\right)^{2},
\end{aligned}
$$

where $h$ is the harmonic number, $g_{0}=1+2 \ln \frac{b}{a}$ is the geometric factor, $Z_{0}$ is the impedance of vacuum, $N_{\mathrm{B}}$ is 
the number of particles per bunch, and $R$ is the mean radius of the synchrotron. Note that the space-charge force has a defocusing effect below transition energy $\left(\cos \phi_{s} \geq 0\right)$ and a focusing effect above the transition energy $\left(\cos \phi_{s} \leq 0\right)$. It causes sudden change of the shape of matched ellipse and thus the mismatch between the beam bunch and the ellipse. Figure 20 shows the rf voltage (RFSUM) and the voltage induced by the space-charge impedance for 12-turn injection. The voltage mismatch due to the space-charge potential can cause post-transition bunch length oscillation.

After passing the nonadiabatic transition-energy region, the particles in the beam bunch start to follow the ellipses of the Hamiltonian torus again. Because the space-charge force above the transition energy is focusing, the bunch ellipse is mismatched to the bucket ellipses. Hence the bunch starts to tumble in the bucket at the rate of synchrotron tune, which causes the rms momentum width of the beam to oscillate at twice the synchrotron tune. Let $\delta_{1}, \delta_{2}$ be the maximum and minimum rms momentum width, which are connected by the matched ellipses [2]

$$
\delta_{2}=\frac{\nu_{s}}{h \eta} \frac{\tilde{\mathcal{A}}}{\delta_{1}},
$$

where $\tilde{\mathcal{A}}=\pi \delta_{1} \phi_{1}$ is the rms phase-space area. The extrema of horizontal beam width are related by

$$
\sigma_{x, \max }^{2}-\sigma_{x, \min }^{2}=D^{2}\left(\delta_{1}^{2}-\delta_{2}^{2}\right) .
$$

We can identify $\hat{\delta}$ in Eq. (13) as $\delta_{1}$. The phase-space area $\mathcal{A}$ (in eV-s) in Eq. (13) is related with the phase-space area $\tilde{\mathcal{A}}$ of Eq. (16) by

$$
\tilde{\mathcal{A}}=\frac{\omega_{0}}{\beta^{2} E} \mathcal{A} .
$$

Combining Eqs. (13), (16), and (17), we can solve for $\delta_{1}$, $\delta_{2}$ and the phase-space area $\mathcal{A}$ from the oscillation mag-

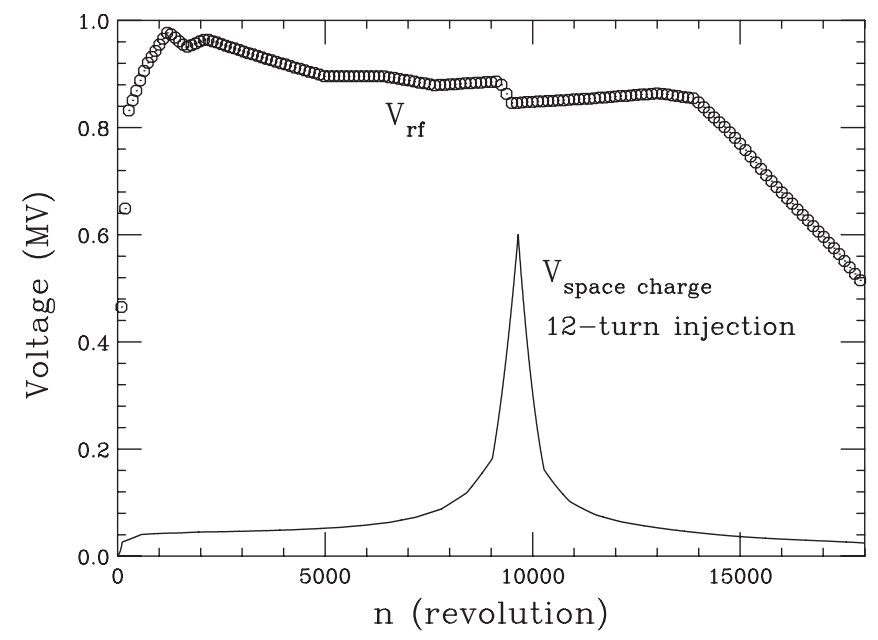

FIG. 20. The effective space-charge voltage $V_{\text {spchg }}$ (solid) and the $V_{\text {rf }}$ in the acceleration cycle. nitude of $\sigma_{x}^{2}$. This is equivalent to the measurement of the quadrupole mode transfer function [17].

The horizontal beam width oscillation can be seen in Fig. 13. We fit oscillation pattern of $\sigma_{x}^{2}$ with

$$
\sigma_{x}^{2}(t)=a+b t+c t^{2}+A \exp (-\alpha t) \cos \left[2 \pi\left(f_{1} t+f_{2} t^{2}\right)+\chi\right]
$$

where $t$ is the revolution number. We apply this fitting model to data from revolution 10501 to revolution 13500 (transition is at revolution 9500, but we avoid the nonadiabatic motion of the first 1000 revolutions after the transition). Examples of fitting curves are shown in Fig. 21.

The oscillatory part of $\sigma_{x}^{2}$ comes essentially from the longitudinal distribution mismatch with the rf bucket. Figure 22 shows the fitted tune parameters $f_{1}$ and $f_{2}$ as a function of the injection turn. The fitted oscillation tune is $f_{1}+2 f_{2} t=0.0065$ at 10500 revolution $(18.6 \mathrm{~ms})$ for 4 turn injection cycle, which is about twice of the synchrotron tune $\nu_{s}=0.0034$ measured from turn-by-turn data at $18.4 \mathrm{~ms}$ with the same intensity [8].

The fitted oscillation amplitude $A$ and the decoherent coefficient $\alpha$ are shown in the bottom plots of Fig. 22. The oscillation amplitude
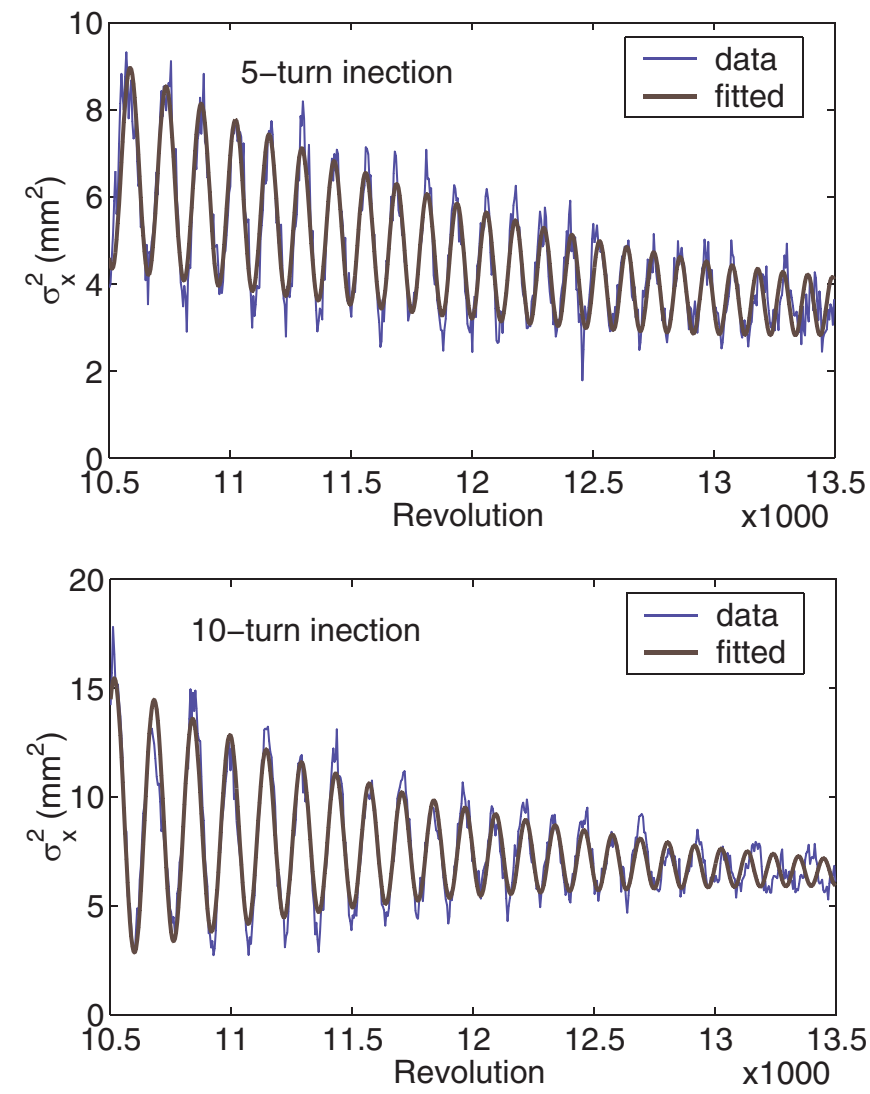

FIG. 21. (Color) Top: Fit $\sigma_{x}^{2}$ to the model of Eq. (19) for 5-turn injection. Bottom: Fit $\sigma_{x}^{2}$ to the model of Eq. (19) for 10-turn injection. 

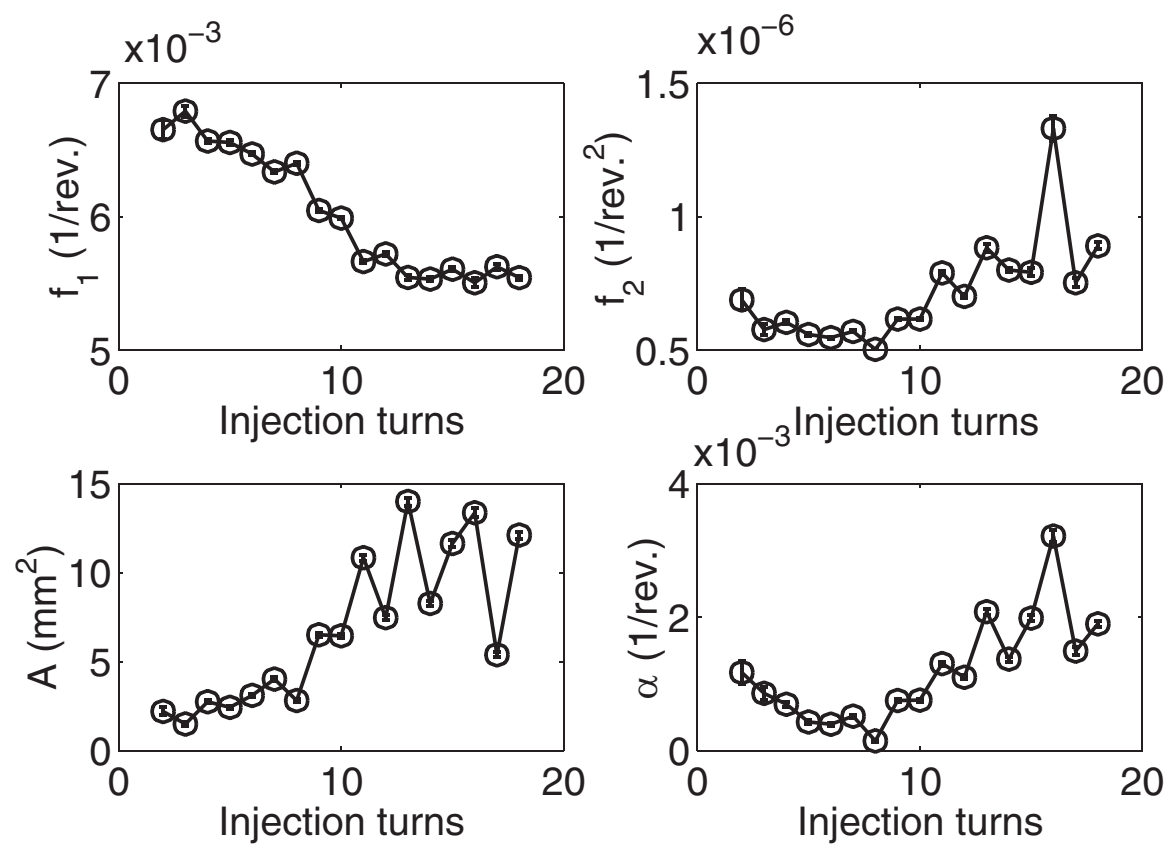

FIG. 22. The top two plots show the fitted post-transition oscillation tune $f_{1}\left(1 /\right.$ revolution) and $f_{2}\left(1 /\right.$ revolution $\left.^{2}\right)$. The oscillation tune is given by $f_{1}+2 f_{2} t$. The bottom plots show the fitted post-transition oscillation amplitude $A$ (mm ${ }^{2}$ ) (bottom left) and the decoherent factor $\alpha$ (1/revolution) (bottom right).

$$
2 A=\sigma_{x, \max }^{2}-\sigma_{x, \min }^{2}
$$

can be used to solve the longitudinal phase-space area. Employing Eqs. (13), (16), and (17), we can selfconsistently solve the mismatched motion. The resulting $\delta_{1}$ and $\delta_{2}$ are shown in Fig. 23. Knowing the maximum $\left(\delta_{1}\right)$ and minimum $\left(\delta_{2}\right)$ of rms momentum width, the average value $\bar{\delta}$ can be calculated

$$
\bar{\delta}_{\text {osci }}=\sqrt{\frac{\delta_{1}^{2}+\delta_{2}^{2}}{2}}
$$

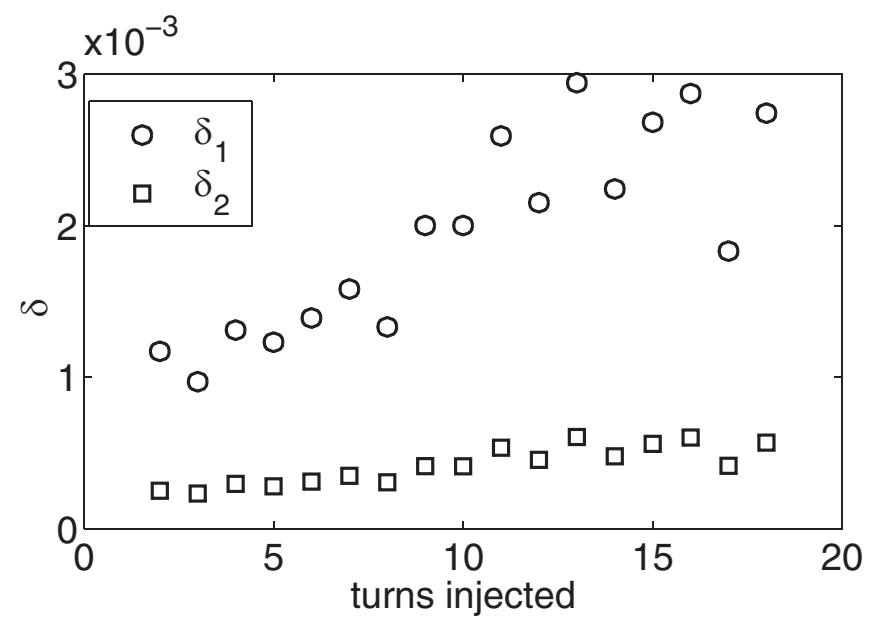

FIG. 23. The deduced mismatched rms momentum spread of the beam $\delta_{1}$ and $\delta_{2}$. where subscript "osci" indicates $\bar{\delta}$ is derived from the oscillation component of $\sigma_{x}^{2}$.

The nonoscillatory part

$$
\sigma_{x, \text { static }}^{2}=a+b t+c t^{2}
$$

is composed of transverse betatron-motion component and the static off-momentum width component. It can be decomposed into the transverse and longitudinal components. Figure 24 shows the fitted parameters for the nonoscillatory part, and the corresponding normalized $\chi^{2}$ normalized to the number of data points for the entire set.

Because the transition energy affects mainly the longitudinal motion, it is reasonable to assume that the transverse emittance will keep growing in the same manner as in the pretransition region. The vertical emittance growth across transition (Fig. 7) suggests the same picture. Thus we have

$$
\sigma_{x, \text { static }}^{2}=\frac{\beta_{x}}{\beta \gamma} \epsilon_{x 0}^{n}\left(1+\alpha_{x} t\right)+D^{2} \bar{\delta}^{2},
$$

where $\epsilon_{x, \text { rms }}^{n}$ is normalized rms emittance and $\alpha_{x}$ is horizontal emittance growth rate. The scaling rule is $\bar{\delta} \sim$ $|\eta|^{-1 / 4} \gamma^{-3 / 4}$, neglecting the orf voltage $V$ factor which is constant in the concerned region. The scaling rule does not include the phase-space dilution from the smearing of the mismatch bunch. By subtracting the predicted transverse component $\beta_{x} \epsilon_{x, \text { rms }}$ from $\sigma_{x \text {,static }}^{2}$, the rms momentum width can also be calculated by

$$
\bar{\delta}_{\text {static }}=\frac{\sqrt{\sigma_{x}^{2}-\beta_{x} \epsilon_{x, \mathrm{rms}}}}{D} .
$$



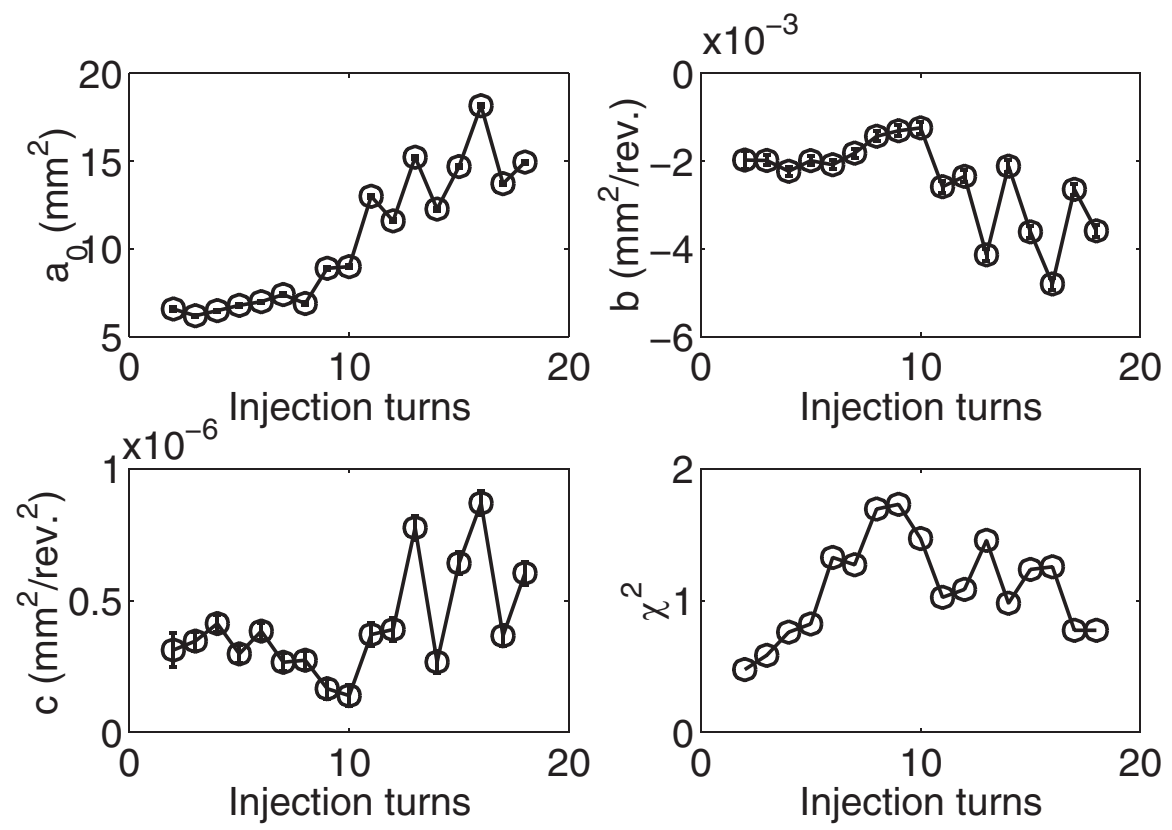

FIG. 24. The fitting parameters of the post-transition horizontal beam size $\sigma_{x}^{2}$. Parameter $a\left(\mathrm{~mm}^{2}\right)$ at top left; parameter $b$ $\left(\mathrm{mm}^{2} /\right.$ revolution) at top right; parameter $c\left(\mathrm{~mm}^{2} /\right.$ revolution $\left.{ }^{2}\right)$ at bottom left; and bottom right: the residual $\chi^{2}$ normalized by noise sigma and number of data points.

Using the growth rate obtained with the pretransition fitting to predict $\epsilon_{x, \text { rms }}$ at revolution 10501 , we have calculated $\bar{\delta}$ for all data sets, which are compared to the results obtained with Eq. (21) in Fig. 25. It is seen that the two methods produce consistent results.

\section{MODELING AND EMITTANCE DILUTION MECHANISMS}

In the last section, we have carried out phenomenological fit for the IPM measurement data at the Fermilab

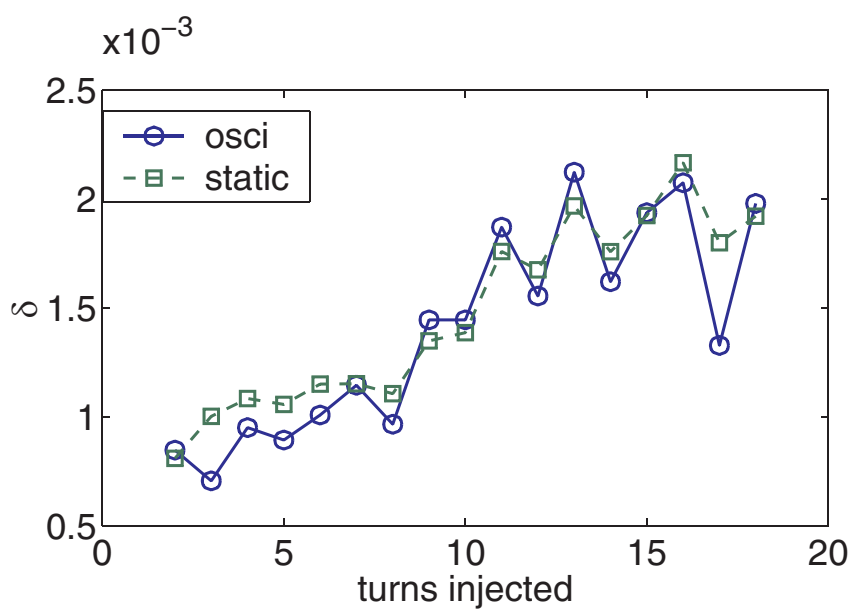

FIG. 25. (Color) The average rms momentum width $\delta(\bar{\delta}$ in the text) obtained with two methods: Eq. (23) ("static") or Eq. (21) (osci).
Booster under various beam intensity levels. Our findings can be summarized as follows:

(1) The normalized vertical emittance starts at about $2 \pi \mathrm{mm} \mathrm{mrad}$ for all intensity levels, i.e., there is no injection phase-space painting. For intensity less than 10-turn injection, the space charge is less important, and the injection efficiency is nearly independent of the intensity (see Fig. 2). This implies that beam loss essentially arises in the longitudinal phase space. Longitudinal phase-space painting with chopped beam may be needed to minimize the beam loss and satisfy the requirement of fast ramping. When the beam intensity is larger than 10-injection turns, the normalized vertical emittance grows rapidly in the first 4000 revolutions. The space-charge force is believed to be the source of the emittance growth.

(2) In the later time of the cycle at $\gamma>2$, the vertical emittance grows linearly with a growth rate of about $1 \pi \mathrm{mm} \mathrm{mrad}$ in $10^{4}$ revolutions. Both the intrabeam scattering and the beam-gas scattering growth rates are too small to explain this linear emittance growth rate.

(3) The horizontal beam width is composed of both the transverse and the longitudinal phase-space distribution of the beam. Making use of the fact that the two components have different momentum scaling rules, we can effectively decompose $\sigma_{x}^{2}$ into betatron and off-momentum beam widths. Such fitting works well for data sets with less than 10-turn injection when the IPM profile calibration error due to space charge is mild [10]. The fitting results yield consistent horizontal normalized emittance and the rms momentum width. Note that the horizontal normalized emittance does not exhibit an explosive growth in the first 
4000 revolutions as observed in the vertical plane. On the other hand, the linear growth rate of the normalized horizontal emittance was also about $0.8 \pi \mathrm{mm} \mathrm{mrad}$ in $10^{4}$ revolutions.

(4) The horizontal beam width starts to oscillate with twice the synchrotron frequency (quadrupole mode) after transition. Such oscillation arises from the mismatch between beam bunch and longitudinal phase-space ellipse. The mismatch was believed to be due to the longitudinal space-charge force which is defocusing before transition and focusing after transition. We extracted the oscillatory and nonoscillatory parts of $\sigma_{x}^{2}$ by fitting it to a model with second-order polynomial plus damped oscillation with linear phase shift rate. The oscillation amplitude of $\sigma_{x}^{2}$ can be used to derive the longitudinal phase-space area. The nonoscillatory part is again separated into horizontal emittance and static (average) rms off-momentum width by assuming that the horizontal normalized emittance grows linearly as in the region before transition. The estimated average rms momentum widths derived from the oscillatory and nonoscillatory parts are consistent. The post-transition bunch length (and beam size) oscillation is a possible cause of beam loss at transition. The bunch shape mismatch may be compensated by rf voltage modulation at a frequency slightly larger than twice the synchrotron frequency [18]. A voltage modulation depth of about $3 \%$ of the total rf cavity voltage, i.e., about $24 \mathrm{kV}$, is sufficient to damp the mismatched oscillation. We have learned that Fermilab Booster has recently implemented the quadrupole-mode damper and has since effectively damped the quadrupole mode oscillation [19].

\section{A. Modeling algorithm}

To understand the phenomena involved in the emittance growth, we construct a multiparticle simulation program to simulate the evolution of beam emittances. As the beam injected into a synchrotron, particles in the synchrotron experience also space-charge force. We track particles including the space-charge force to extract the essential mechanisms of emittance growth for the Fermilab Booster. We will describe our numerical-simulation algorithm in the following subsections.

\section{Energy gain in rf cavities}

Since rf cavities at the Fermilab Booster are located in dispersive locations, the beam is constantly and coherently excited by the energy gain at about $400 \mathrm{keV}$ per revolution. Can this coherent beam excitation combined with beam decoherence produce the observed emittance growth?

Fermilab Booster is a fast-ramping accelerator. Semiadiabatic capture is applied in the $\mathrm{H}^{-}$injection scheme. The rf voltage is ramped from a small number to about $900 \mathrm{kV}$ to provide fast cycling of $15 \mathrm{~Hz}$. The rf voltage $V_{\mathrm{rf}}$ and the synchronous phase $\phi_{s}$ were recorded, and employed for this tracking. Energy gain in each revolution is $V_{\mathrm{rf}} \sin \phi_{s}$, which is equally distributed in all cavities located in each particular long straight section with rf cavities.

We assume that the dipole magnets are ramped linearly, while the beam receives energy at cavities with energy $u$ in each cavity that is located in nonzero dispersive region. The horizontal position of a particle is given by

$$
x=x_{\beta}+D \frac{\Delta E}{\beta^{2} E}, \quad x^{\prime}=x_{\beta}^{\prime}+D^{\prime} \frac{\Delta E}{\beta^{2} E},
$$

where $\left(x_{\beta}, x_{\beta}^{\prime}\right)$ are the betatron coordinates, and $\left(D, D^{\prime}\right)$ are the dispersion function. Each particle gains the same amount of energy in rf cavities. As the energy of a particle is gained in a cavity, the changes of the betatron coordinates are

$$
\Delta x_{\beta}=-D \frac{u}{\beta^{2} E}, \quad \Delta x_{\beta}^{\prime}=-D^{\prime} \frac{u}{\beta^{2} E},
$$

where $u$ is the energy gain at the cavity. The centroid of the beam is constantly and coherently excited by the betatron excitation.

We define the fractional momentum deviation between the beam momentum $p_{\text {beam }}$ and the "magnet-momentum" $p_{\text {mag }}$ (the momentum associated with the magnetic field) as

$$
\frac{\Delta p}{p} \equiv \frac{p_{\text {beam }}-p_{\text {mag }}}{p_{\text {beam }}} .
$$

The top plot of Fig. 26 shows that the beam momentum is
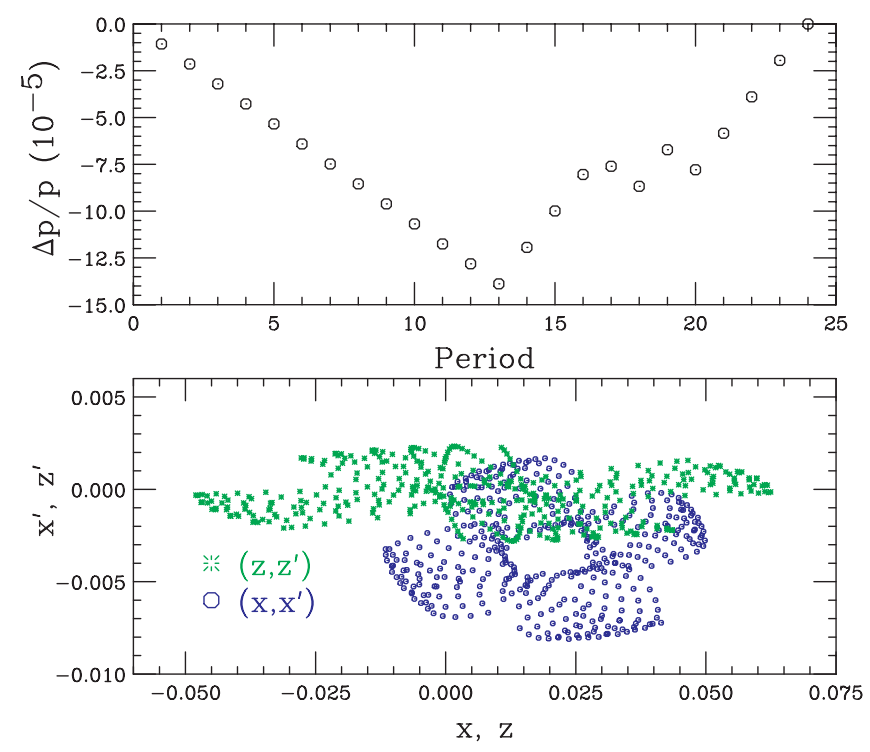

FIG. 26. (Color) Top: The fractional momentum deviation of the beam with respect to the momentum associated with the dipole magnetic field. The energy gain is $400 \mathrm{keV}$ per revolution with beam momentum $p_{0}=1.78 \mathrm{GeV} / c$ in this example. The actual energy gain in our simulation program per revolution is obtained from the RFSUM data of the booster operation. Bottom: The coherent betatron motion of the beam excited by the energy gain in each cavity. A linear coupling with a focal length of about 200 $\mathrm{m}$ is included in attaining the coherent betatron motion in the vertical plane. The units for $(x, z)$ and $\left(x^{\prime}, z^{\prime}\right)$ are $\mathrm{mm}$ and $\mathrm{mrad}$, respectively. 
constantly oscillating against the momentum associated with the magnetic field. The bottom plot shows the betatron motion of the centroid of the beam for the first 400 revolutions with linear coupling at a coupling strength of $\left(\int a_{1} d s\right)=$ $0.005 \mathrm{~m}^{-1}$.

\section{FODO cells and random linear errors}

The booster is made of 24 combined function FODO cells. Particle transport in each FODO cell is carried out by the transfer matrix from D to $\mathrm{F}$ with

$$
M_{\mathrm{D} \rightarrow \mathrm{F}}=\left(\begin{array}{cccc}
\sqrt{\frac{\beta_{x, \mathrm{~F}}}{\beta_{x, \mathrm{D}}}} \cos \psi_{x} & \sqrt{\beta_{x, \mathrm{~F}} \beta_{x, \mathrm{D}}} \sin \psi_{x} & 0 & 0 \\
-\frac{1}{\sqrt{\beta_{x, \mathrm{~F}} \beta_{x, \mathrm{D}}}} \sin \psi_{x} & \sqrt{\frac{\beta_{x, \mathrm{D}}}{\beta_{x, \mathrm{~F}}}} \cos \psi_{x} & 0 & 0 \\
0 & 0 & \sqrt{\frac{\beta_{z, \mathrm{~F}}}{\beta_{z, \mathrm{D}}}} \cos \psi_{z} & \sqrt{\beta_{z, \mathrm{~F}} \beta_{z, \mathrm{D}}} \sin \psi_{z} \\
0 & 0 & -\frac{1}{\sqrt{\beta_{z, \mathrm{~F}} \beta_{z, \mathrm{D}}}} \sin \psi_{z} & \sqrt{\frac{\beta_{z, \mathrm{D}}}{\beta_{z, \mathrm{~F}}}} \cos \psi_{z}
\end{array}\right),
$$

where $\psi_{x, z}=2 \pi \nu_{x, z} / 48$ is the phase advance in a half-cell. We have assumed $\alpha_{x, F}=0$ and $\alpha_{z, F}=0$.

Similarly, the transfer matrix from the F back to D is

$$
M_{\mathrm{F} \rightarrow \mathrm{D}}=\left(\begin{array}{cccc}
\sqrt{\frac{\beta_{x, \mathrm{D}}}{\beta_{x, \mathrm{~F}}}} \cos \psi_{x} & \sqrt{\beta_{x, \mathrm{~F}} \beta_{x, \mathrm{D}}} \sin \psi_{x} & 0 & 0 \\
-\frac{1}{\sqrt{\beta_{x, \mathrm{~F}} \beta_{x, \mathrm{D}}}} \sin \psi_{x} & \sqrt{\frac{\beta_{x, \mathrm{~F}}}{\beta_{x, \mathrm{D}}}} \cos \psi_{x} & 0 & 0 \\
0 & 0 & \sqrt{\frac{\beta_{z, \mathrm{D}}}{\beta_{z, \mathrm{~F}}}} \cos \psi_{z} & \sqrt{\beta_{z, \mathrm{~F}} \beta_{z, \mathrm{D}}} \sin \psi_{z} \\
0 & 0 & -\frac{1}{\sqrt{\beta_{z, \mathrm{~F}} \beta_{z, \mathrm{D}}}} \sin \psi_{z} & \sqrt{\frac{\beta_{z, \mathrm{~F}}}{\beta_{z, \mathrm{D}}}} \cos \psi_{z}
\end{array}\right) .
$$

The parameters for the betatron amplitude and dispersion functions are $\beta_{x, \mathrm{D}}=6.3 \mathrm{~m}, \beta_{z, \mathrm{D}}=21.4 \mathrm{~m}, \beta_{x, \mathrm{~F}}=40 \mathrm{~m}$, $\beta_{z, \mathrm{~F}}=8.3 \mathrm{~m}, \quad D_{x, \mathrm{D}}=2.54 \mathrm{~m}, \quad$ and $D_{x, \mathrm{~F}}=4.5 \mathrm{~m}$. Figure 27 shows the betatron tunes during the $33 \mathrm{~ms}$ ramping cycle.

Systematic sextupoles and small random dipole, quadrupole and skew-quadrupole errors are introduced as kicks to each particle:

$$
\begin{gathered}
x^{\prime \prime}+K_{x}(s) x=b_{0}(s)+b_{1}(s) x+a_{1}(s) z-\frac{1}{2} b_{2}(s)\left(x^{2}-z^{2}\right), \\
z^{\prime \prime}+K_{z}(s) z=-a_{0}(s)-b_{1}(s) z+a_{1}(s) x+b_{2}(s) x z .
\end{gathered}
$$

The dipole components $b_{0}$ and $a_{0}$ are applied to each particle with a random seed to simulate the closed-orbit misinjection. The random quadrupole error is intended to create half-integer stop band in order to test the importance of the envelope dynamics. The quadrupole component $b_{1}(s)$ is generated by a random seed with zero tune shift, i.e., $\oint \beta_{x}(s) b_{1}(s) d s=0$ and $\oint \beta_{z}(s) b_{1}(s) d s=0$. The random skew quadrupoles take into account the quadrupole roll and the vertical closed-orbit error in sextupoles. All random numbers are generated by random seeds with uniform distribution from -1 to +1 . The magnitude of these random linear error fields is controlled by an amplitude, i.e.,

$$
\begin{aligned}
& A_{b 0}(n)=A_{b 0}(0) \exp \left\{-\frac{n}{N_{\text {dipole }}}\right\}, \\
& A_{a 0}(n)=A_{a 0}(0) \exp \left\{-\frac{n}{N_{\text {dipole }}}\right\}, \\
& A_{b 1}(n)=A_{b 1}(0) \exp \left\{-\frac{n}{N_{\text {quad }}}\right\}, \\
& A_{a 1}(n)=A_{a 1}(0) \exp \left\{-\frac{n}{N_{\text {quad }}}\right\}
\end{aligned}
$$

The damping of the dipole kicks is intended to take into account the decoherence effect associated with the initial closed-orbit error. The damping of quadrupole kicks takes into account the fact that the particle orbit will eventually evolve into the matched ellipse of the accelerator. The effect of dipole error is most important during the injection, while the effect of quadrupole error requires a longer time to settle into matched beam ellipse. We choose $N_{\text {dipole }}=3000$, and $N_{\text {quad }}=4000$.

The sextupole is inherent to all combined function dipoles. They are introduced as a thin-lens kick at the end of each half-cell. The sextupole strengths are $\left(\int b_{2} d s\right)=$ $-0.0173 \mathrm{~m}^{-2}$ and $-0.263 \mathrm{~m}^{-2}$ for focusing and defocusing dipoles, respectively, for each of the half-FODO cell. We also include sextupoles in the short and long straight 


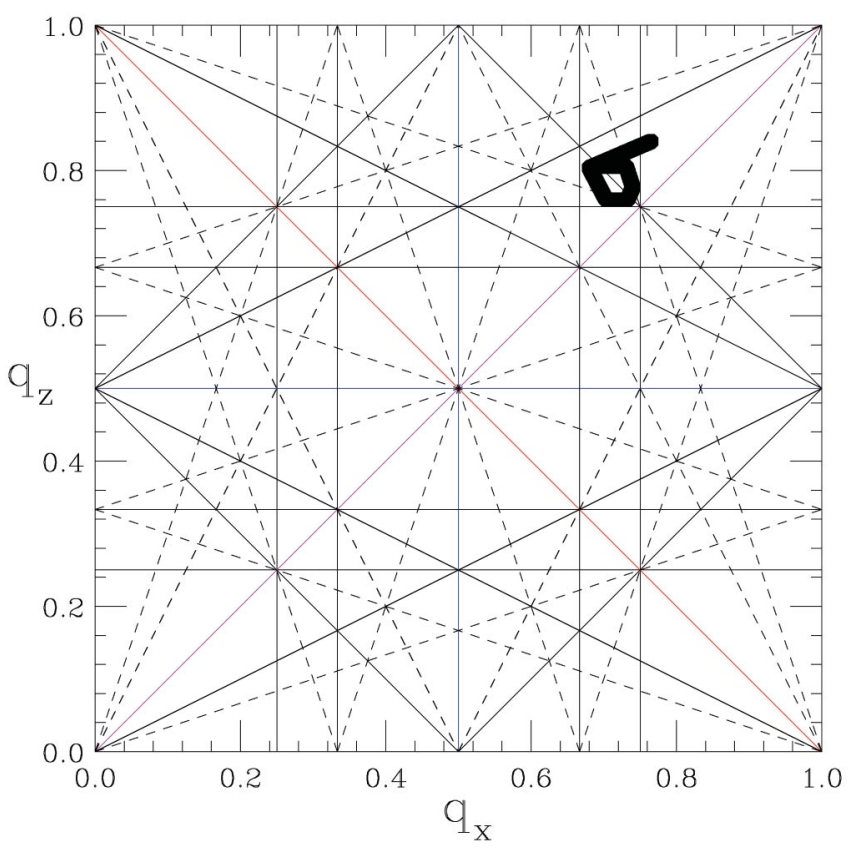

FIG. 27. (Color) The fractional part of the betatron tune during the entire ramping cycle from injection at $400 \mathrm{MeV}$ to the top energy at 8. GeV (see Fig. 9 in [8]).

sections to explore their effects on the emittance growth. However, we find that their effects on emittances are small.

\section{Space-charge effects}

We assume a Gaussian charge distribution:

$$
\rho(x, z)=\frac{N e}{2 \pi \sigma_{x} \sigma_{z}} \exp \left\{-\frac{x^{2}}{2 \sigma_{x}^{2}}-\frac{z^{2}}{2 \sigma_{z}^{2}}\right\}
$$

where $N e$ is the charge per unit length and $\sigma_{x}$ and $\sigma_{z}$ are the horizontal and vertical $\mathrm{rms}$ beam radii. The horizontal rms beam radius is composed of both the betatron and offmomentum width contributions. For a Gaussian charge distribution, the transverse space-charge potential is [20]

$$
\begin{aligned}
V(x, z)= & \frac{N r_{0}}{\beta^{2} \gamma^{3}} \int_{0}^{\infty} \frac{-1+\exp \left\{-\frac{x^{2}}{2 \sigma_{x}^{2}+t}-\frac{z^{2}}{2 \sigma_{z}^{2}+t}\right\}}{\sqrt{\left(2 \sigma_{x}^{2}+t\right)\left(2 \sigma_{z}^{2}+t\right)}} d t \\
\approx & -\frac{N r_{0}}{\beta^{2} \gamma^{3}}\left\{\left(\frac{x^{2}}{\sigma_{x}\left(\sigma_{x}+\sigma_{z}\right)}+\frac{z^{2}}{\sigma_{z}\left(\sigma_{x}+\sigma_{z}\right)}\right)\right. \\
& -\frac{N r_{0}}{4 \beta^{2} \gamma^{3} \sigma_{x}^{2}\left(\sigma_{x}+\sigma_{z}\right)^{2}}\left(\frac{2+R}{3} x^{4}+\frac{2}{R} x^{2} z^{2}\right. \\
& \left.\left.+\frac{1+2 R}{3 R^{3}} z^{4}\right)+\cdots\right\},
\end{aligned}
$$

where the singularity at $x=z=0$ is removed by the addition of the -1 term in the numerator of the integrand.

Because of the space-charge potential, each particle experiences a space-charge kick given by

$$
\begin{aligned}
& \Delta x^{\prime}=-\frac{\partial V}{\partial x} \ell \approx \frac{2 N r_{0} \ell}{\beta^{2} \gamma^{3} \sigma_{x}\left(\sigma_{x}+\sigma_{z}\right)} x \exp \left\{-\frac{x^{2}+z^{2}}{\left(\sigma+\sigma_{z}\right)^{2}}\right\}, \\
& \Delta z^{\prime}=-\frac{\partial V}{\partial z} \ell \approx \frac{2 N r_{0} \ell}{\beta^{2} \gamma^{3} \sigma_{z}\left(\sigma_{x}+\sigma_{z}\right)} z \exp \left\{-\frac{x^{2}+z^{2}}{\left(\sigma+\sigma_{z}\right)^{2}}\right\}
\end{aligned}
$$

where $\ell$ is the length of the half-cell. The form in Eq. (36) is obtained by using the space-charge potential of Eq. (34) up to the second-order expansion in round beam geometry, and exponentiated to produce zero tune shift for large amplitude particles. The space-charge force is approximated by 48 localized kicks per revolution. The rms beam radii calculated from the multiparticle phase-space distribution is used for space-charge kicks in the next revolution. In order to test many conditions, we will carried out non-self-consistent multiparticle simulation based on the potential model, i.e., the space-charge force is modified by the rms radii, but remains in the Gaussian-potential form. Although the distribution function may become non-Gaussian, our space-charge kick remains in the Gaussian beam approximation.

\section{Betatron tunes and the rms beam-momentum spread}

The betatron tunes have been measured during the ramping cycle as shown in Fig. 9 of Ref. [8] (see also Fig. 27). The rf voltage, the synchronous phase, and the bunch length during the ramping cycle have also been measured. In our simulation, we use interpolation between the measured data in a ramping cycle.

We assume that the (95\%) longitudinal bunch area to be $0.08 \mathrm{eV}$-s. After the transition energy, the rms momentum width begins mismatch oscillation. At revolution $n>n_{t}=$ 9600 , the rms off-momentum width $\delta_{\text {rms }}(n)$ is

$$
\begin{aligned}
\delta_{\mathrm{rms}}(n)= & \delta_{\mathrm{rms}, 0} B_{f}(n)\left\{1+\left(G_{\delta}-1\right)\right. \\
& \left.\times\left(1-\exp \left[-\alpha_{\delta}\left(n-n_{t}\right)\right]\right)\right\} \\
& \times\left\{1+A_{\delta} \exp \left[-\alpha_{\delta}\left(n-n_{t}\right)\right]\right. \\
& \left.\times \sin \left[2 \pi\left(n-n_{t}\right) f\right]\right\} .
\end{aligned}
$$

Here $B_{f}(n)$ is the bunching factor, $\delta_{\mathrm{rms}, 0} \approx 3.0 \times 10^{-4}$ is the initial rms off-momentum width, $G_{\delta}$ is the momentum width growth factor, $\alpha_{\delta}$ is the decoherent constant, $A_{\delta}$ is the amplitude of bunch shape oscillation, and $f$ is the oscillation tune. In our simulation, we choose $n_{t}=9600$, $G_{\delta}=2.0, A_{\delta}=0.5, f=1 / 150$ (1/revolution), and $\alpha_{\delta}=$ $1 /(15 \times 150)$.

The focusing strength of quadrupoles in the Fermilab Booster is about $0.33 \mathrm{~m}^{-1}$. Thus the random quadrupole strength is expected to be about $3.0 \times 10^{-4} \mathrm{~m}^{-1}$. The dipole bending strength for each half-FODO cell is about $0.131 \mathrm{rad}$. The amplitude of dipole errors is estimated to be about $2.0 \times 10^{-5}$ radian in the horizontal plane and $7.5 \times$ 
$10^{-5}$ in the vertical plane. The horizontal dipole field error corresponds to about $1.5 \times 10^{-4}$ error in the main dipole field, while the vertical random dipole error field corresponds to about $0.6 \mathrm{mrad}$ of dipole roll. Closed-orbit oscillations are evidently visible in the profile data of Fig. 3 for the first 4000 revolutions. The random skewquadrupole strength can be produced by the vertical closed-orbit deviation in sextupole and by the quadrupole roll. We assume an amplitude of $3.5 \times 10^{-3} \mathrm{~m}^{-1}$ for the random skew quadrupole. This is equivalent to an amplitude of vertical closed orbit of the order of $1.0 \mathrm{~cm}$.

To check our program, we first examine the emittance evolution in the presence of linear dipole and quadrupole errors without space-charge force and sextupole nonlinearity. Figure 28 shows the rms beam radii and beam emittances at the defocusing quadrupole location. The vertical beam width is adiabatically damped, and the horizontal beam width shows the characteristic off-momentum dependence. However, the horizontal and vertical emittances are constant in the absence of space-charge force and sextupole nonlinearity.

\section{B. Results of numerical simulations}

In this section, we will examine the effect of each factor of our algorithm on the emittance growth.

\section{Effect of the half-integer stop band}

The half-integer stop band has been considered as one of the main sources on emittance growth since 1960s [1,2]. When the tunes of the beam envelope function reach an integer, the emittance may grow and beam loss may occur. In our model, the random quadrupole field is introduced as a thin-lens kick in each half-cell with random seeds, which produce a zero betatron tune shift.

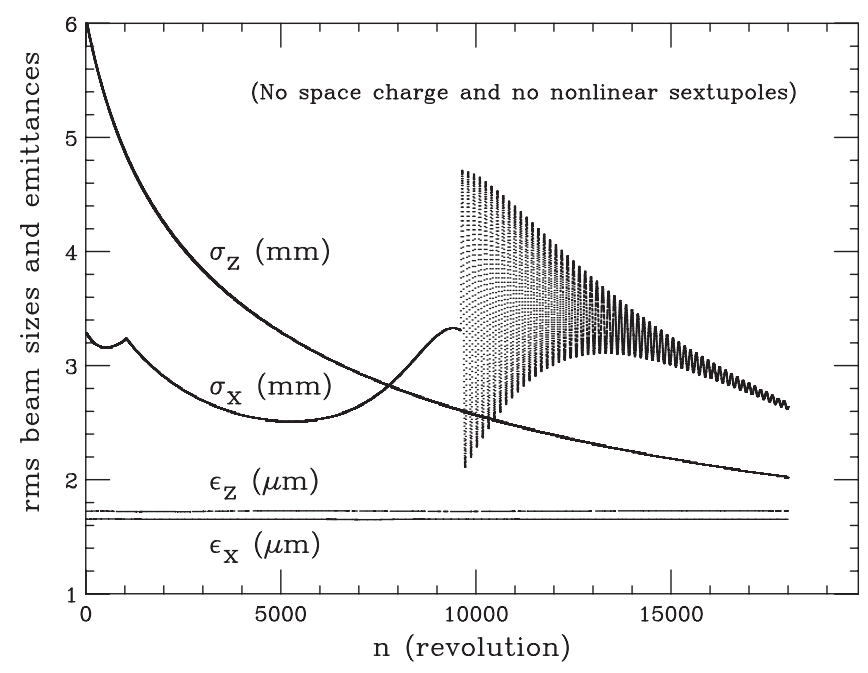

FIG. 28. The emittance is not affected by the linear errors and the coherent beam oscillations due to rf cavities located in dispersive locations.
We first consider only the linear space-charge kick. The existence of the random quadrupole error will produce betatron mismatch. The effect is particularly important if the betatron envelope tune is depressed toward the halfinteger stop band. Figure 29 shows the initial (black dots) and the final (red dots at $n=11000$ revolutions) phasespace distributions for 12-turn injection with the amplitude of random quadrupole amplitude at $A_{b 1}(0)=4.0 \times$ $10^{-4} \mathrm{~m}^{-1}$ (top plots) and $A_{b 1}(0)=4.0 \times 10^{-3} \mathrm{~m}^{-1}$ (bottom) of Eq. (32).

In the presence of the linear random quadrupole error, the phase-space distribution is altered by the linear spacecharge force while the phase-space areas (or emittances) remain constant in these cases. Emittance dilution occurs only when nonlinear forces such as sextupole and higher order multipoles are included. The nonlinearity introduces betatron detuning that gives rise to decoherence in the betatron motion.

We now explore the half-integer stop band including nonlinear decoherence. We introduce a random quadrupole distribution such that the stop band widths are about 0 and 0.1 at harmonics 12 and 13 , respectively. The corresponding amplitude of random quadrupole field error is $40 \times$ $10^{-4} \mathrm{~m}^{-1}$, i.e., about $1.3 \%$ of the main quadrupole field. We turn off all random dipole and skew-quadrupole field
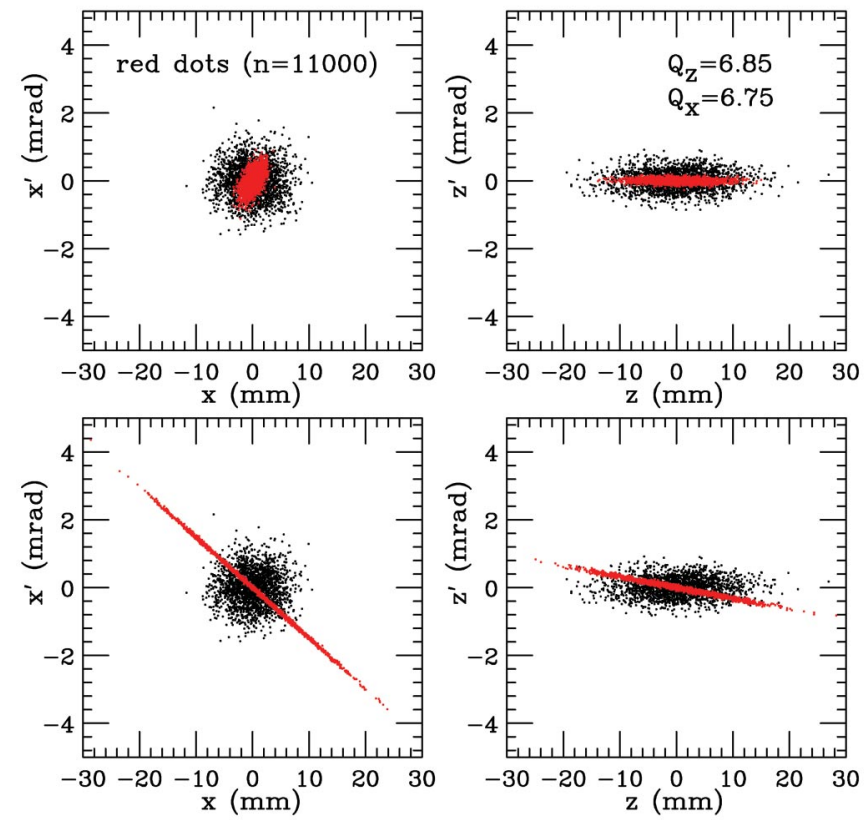

FIG. 29. (Color) The beam phase-space distribution for the initial (black dots) and the final (red dots at $n=$ 11000 revolution or a kinetic energy of $5.0 \mathrm{GeV}$ ) distributions for a beam with 12-turn injection. Top: The initial (black dots) and the final (red dots at $n=11000$ revolution) phase-space plots $\left(x, x^{\prime}\right)$ and $\left(z, z^{\prime}\right)$ for the $A_{b 1}=4.0 \times 10^{-4} \mathrm{~m}^{-1}$. Bottom: The same as that of the top plot except the amplitude of the random quadrupole error is $A_{b 1}=4.0 \times 10^{-3} \mathrm{~m}^{-1}$. 
errors so that we examine only the random quadrupole field error.

We fix the vertical betatron tune at $Q_{z}=6.95$ and vary the horizontal betatron tune $Q_{x}$ from 6 to 7 . Since the bare vertical tune is far away from the stop band by choice, the vertical emittance is constant in all cases. Since the horizontal tune is varied across the stop band 13, and approaches a smaller stop band at harmonic 12, we expect to see large horizontal emittance change during this process. The top plot of Fig. 30 shows the final horizontal emittance as a function of the bare horizontal tune for a beam with 12-turn injection. Because of the nonlinear decoherence, the perturbation in the envelope function gives actual emittance dilution shown in Fig. 30. The horizontal emittance is affected by the stop band in the presence of space charge. When the space-charge force is turned off, the emittance is much less affected by the presence of the half-integer stop band shown as symbols of X's. The bottom plot shows the horizontal Laslett-space-charge tune shift during the ramping process for a small amplitude particle.

The space-charge effect is a self-adjusting process. When the emittance increases or beam loss occurs, the corresponding space-charge tune shift is reduced. Thus in the ramping cycle, the linear space-charge tune shifts may be different in each machine condition. When the betatron tune is sufficiently near the stop band, the beam distribution is rearranged by depleting the particle in the center core, and the distribution may become a hollow ring in phase space. However, we also note that the actual random quadrupole error is expected to be 10 times smaller than those used in this calculation. With the expected gradient error of the order of $4 \times 10^{-4} \mathrm{~m}^{-1}$, we find little emittance growth, shown as red points in Fig. 30. We have carried out half-integer stop band compensation experiments at the Fermilab Booster and have indeed found that the effect of half-integer stop band on the beam emittance is small [21].

\section{Effects of the Montague resonance}

Montague resonance has been extensively studied and considered as one of major sources on emittance growth [5]. In order to investigate the effect of Montague resonance at $2 \nu_{x}-2 \nu_{z}=$ integer resonance, we turn off all linear random errors while retaining only the systematic sextupole fields in dipoles. To investigate the effect of
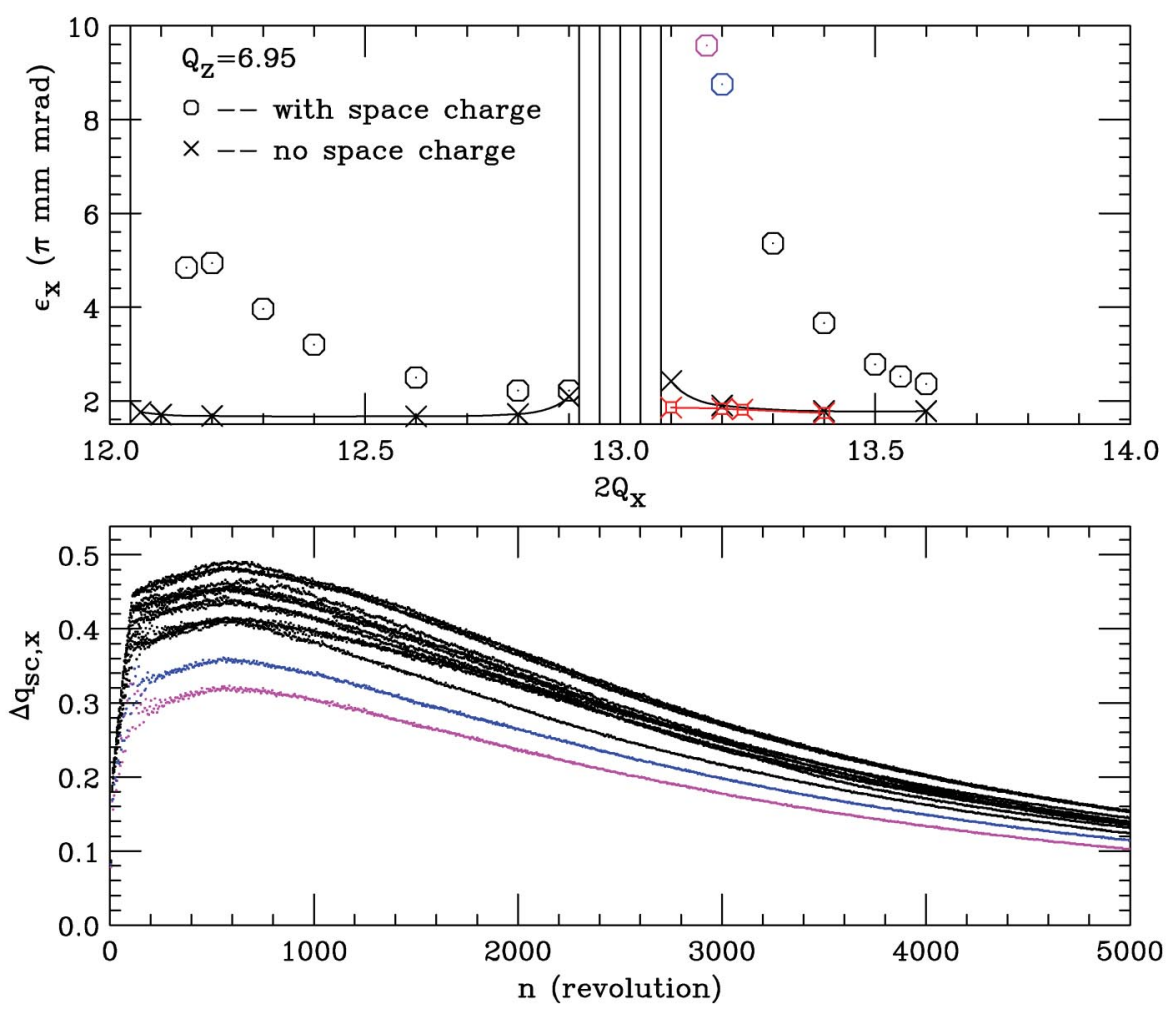

FIG. 30. (Color) Top: The final emittance for a beam with 12-turn injection under the influence of the stop band at harmonics 12 and 13. The stop band width at harmonic 12 is intentionally made small by the choice of a random seed. Bottom: the magnitude of the space-charge tune shift for all cases shown in the top plot. The blue and magenta colors correspond to the large emittance growths shown in the top plots. In these two cases, the beam distribution in the horizontal phase space becomes hollow. Note that the space charge is a self-adjusting process. When a large space-charge tune shift leads to resonance overlapping, the emittance increases so that the space-charge tune shift avoids the resonance overlap. 

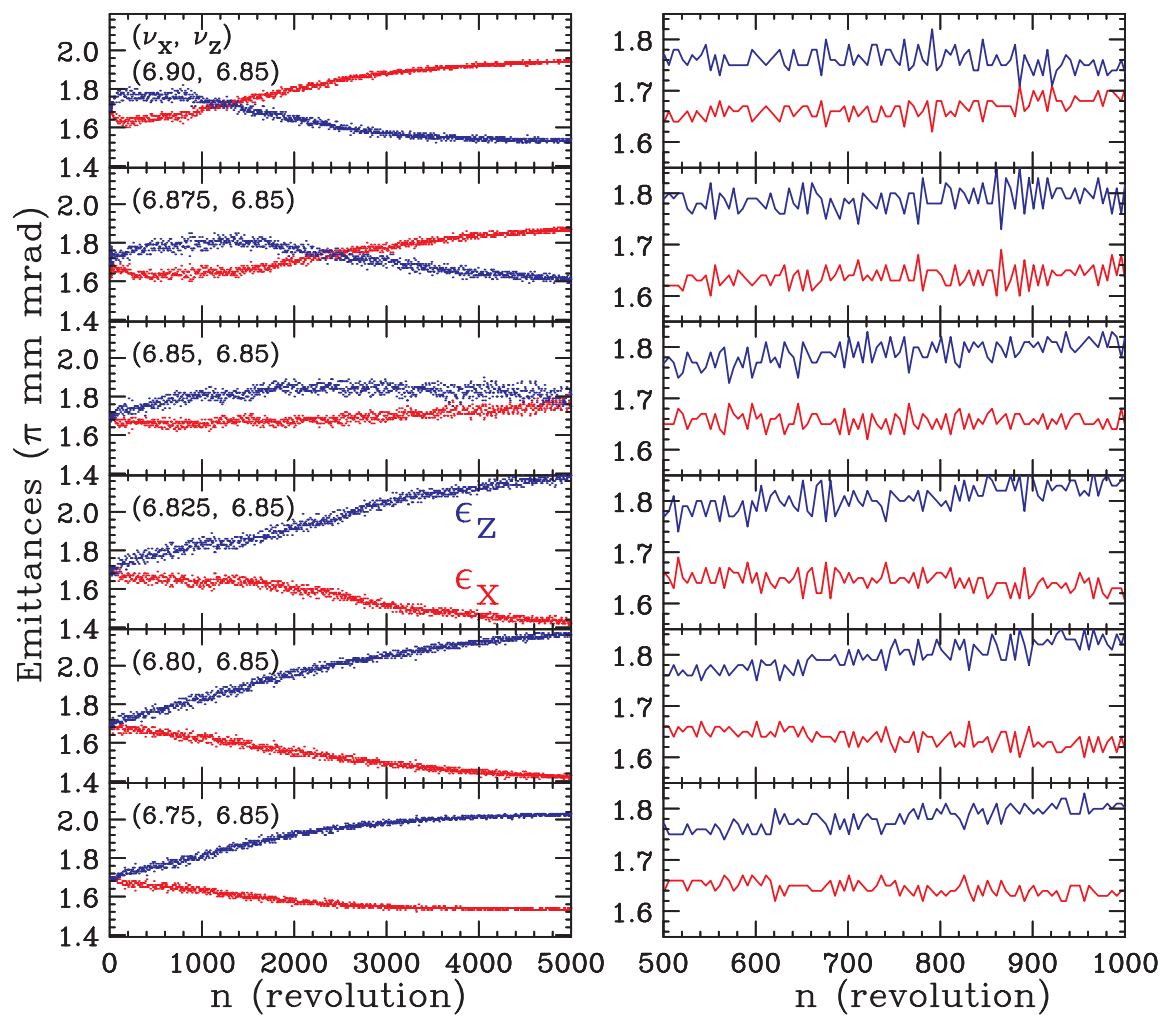

FIG. 31. (Color) The emittance evolution for a beam with 12-turn injection when only the space-charge force is included in the calculation. The horizontal betatron tune is varied across the vertical betatron tune to examine the effect of Montague resonance. The right plots zoom in on revolution 500 to 1000 to see the details of the emittance exchange.

coupling, we also fix the vertical tune at $\nu_{z}=6.85$, and change the horizontal tune from 6.75 to 6.90 . Figure 31 shows the effects of Montague resonance as the horizontal tune is varied across the vertical betatron tune.

The initial growth of the vertical emittance in Fig. 31 arises from the phase-space mismatch. When the horizontal and vertical betatron tunes are equal, two emittances are fully coupled, and the emittances tend to be equal. When the horizontal and vertical betatron tunes are not equal, the emittances are exchanged so that the space-charge tune spread moves away from the Montague resonance line. Although the Montague resonance can cause emittance oscillation and exchange, its effect on the emittance growth is small.

\section{Effect of the linear skew quadrupoles}

Vertical closed-orbit deviation in sextupoles and quadrupole roll can induce skew-quadrupole field. Random skew-quadrupole kicks are applied at each half-cell. The amplitude of the random skew-quadrupole field is estimated to be about $3.50 \times 10^{-3} \mathrm{~m}^{-1}$, or a focal length of about $300 \mathrm{~m}$. This corresponds to an amplitude of vertical closed-orbit error of the order of $1.0 \mathrm{~cm}$.

The random skew-quadrupole field is much harder to investigate because it may mix up with the Montague resonance. We explore the effect by varying strengths of the random skew quadrupoles with an identical random number seed. Figure 32 shows the emittances vs the horizontal tune $Q_{x}$, while the vertical tune is fixed at $Q_{z}=$ 6.85. The random skew quadrupoles are applied to every half-FODO cell with amplitudes $0,3.5 \times 10^{-4} \mathrm{~m}^{-1}, 14 \times$ $10^{-4} \mathrm{~m}^{-1}, 35 \times 10^{-4} \mathrm{~m}^{-1}$, respectively.

The blue lines (square symbols) are emittances for the calculation without random skew-quadrupole field. Note that the Montague resonance is most important when the horizontal and vertical tunes are equal. The emittances are shown to be nearly equal in Fig. 32 when this condition is met. On the other hand, when the horizontal betatron tune approaches any integer, particle encounters coherent kicks and the horizontal emittance becomes very large. The phase-space distribution becomes hollow in the horizontal phase space.

When random skew-quadrupole kicks are introduced in the accelerator, it introduces linear-coupling resonances $\nu_{x} \pm \nu_{z}=$ integer resonances. When the skew-quadrupole strength is small, e.g., $g_{\mathrm{sq}}=3.5 \times 10^{-4} \mathrm{~m}^{-1}$ as shown as an X symbol (green lines) in Fig. 32, the effect does not differ much from that of no skew quadrupole. When the amplitude of skew-quadrupole strength is increased to $14 \times 10^{-4} \mathrm{~m}^{-1}$, shown as black lines in Fig. 32, both the linear-coupling difference and sum resonances becomes important. When the amplitude of skew quadrupoles is 


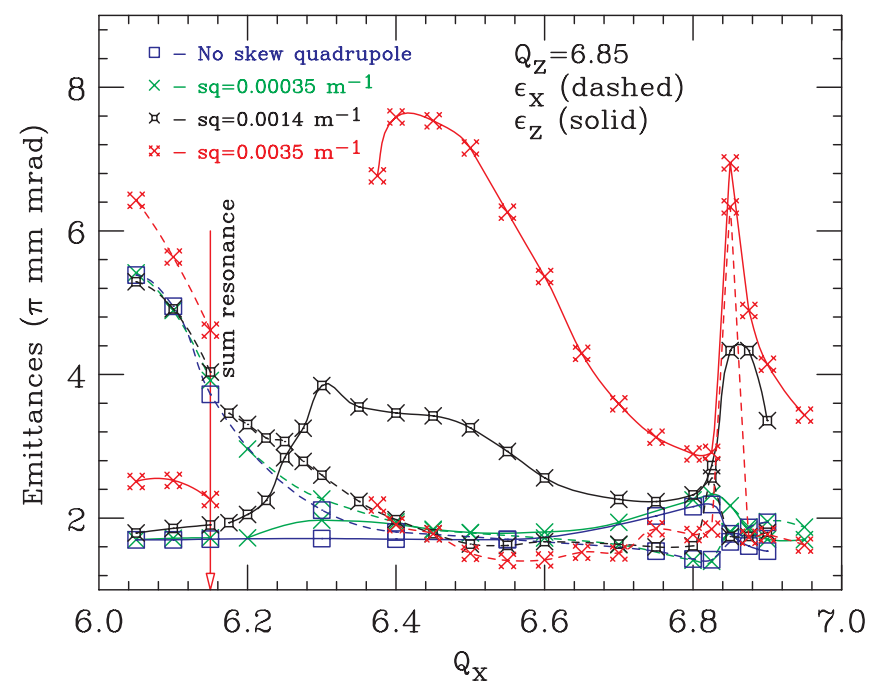

FIG. 32. (Color) The emittance evolution for a beam with 12turn injection when the space-charge force and random skewquadrupole kicks are included. The importance of the linearcoupling (sum and difference) resonances are clearly visible when the amplitude of skew quadrupole is large. Note that the random skew-quadrupole error also introduces stop band width to integer resonances.

$35 \times 10^{-4}$, as shown in the red lines in Fig. 32, the linearcoupling resonances becomes extremely strong. Substantial beam loss will occur when $Q_{x}$ falls in the width of the stop band, i.e., $6.15<Q_{x}<6.35$. In particular, the sum resonance $\nu_{x}+\nu_{z}=$ integer produces a very large vertical emittance growth even when the resonance is still far away. Figure 33 shows the initial (black) and final (red) phase-space plot for the case with betatron tunes at $Q_{x}=$ 6.30 and $Q_{z}=6.85$. We note that the distribution function
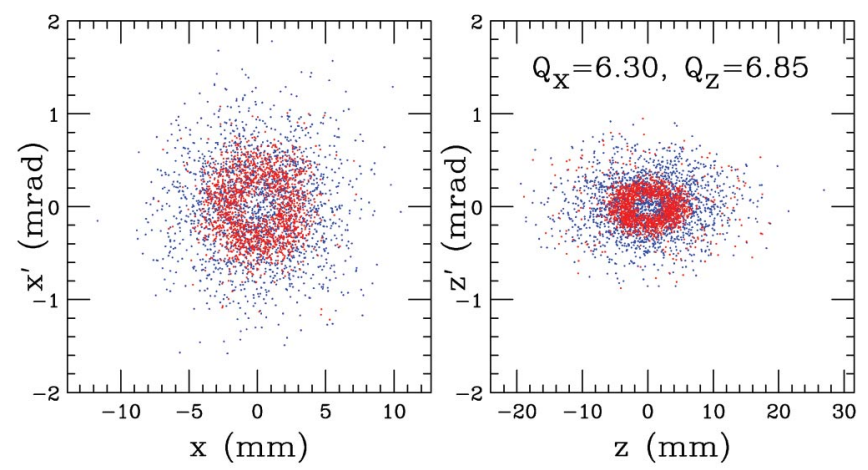

FIG. 33. (Color) The horizontal (left plot) and vertical (right plot) phase-space distributions for a beam with 12-turn injection for skew-quadrupole amplitude $0.0014 \mathrm{~m}^{-1}$. The initial phasespace points, shown in blue dots, have normalized emittances of about $1.7 \pi \mathrm{mm} \mathrm{mrad}$ on both planes. The final phase-space distributions, shown in red at 6000 revolution, have normalized emittances 2.6 and $3.8 \pi \mathrm{mm}$ mrad in the horizontal and vertical planes, respectively. Note that the phase-space distribution becomes hollow in both the horizontal and vertical planes. becomes hollow rings in both the horizontal and vertical phase spaces.

To examine the stop band width of the sum resonance in the presence of space-charge tune spread, we examine the emittance growth vs the beam intensity. Figure 34 shows the horizontal (solid line) and vertical (dashed line) emittances as a function of the horizontal betatron tune $Q_{x}$ for a fixed vertical betatron tune at $Q_{z}=6.85$. The intensity is varied from 1-turn injection to 11-turn injection. We note that the stop band width increases with the beam intensity. Substantial beam loss occurs when the bare tunes fall within the stop band.

The skew quadrupole generates a surprising emittance growth mechanism for the difference coupling resonance at $\nu_{x}-\nu_{z}=$ integer. Figures 32 and 34 show that the emittances grow together when the horizontal and vertical tunes are equal. This is counterintuitive. We expect that the sum of the horizontal and vertical emittances should be constant at a linear difference resonance. The reason of the emittance growth for the linear-coupling resonance is the phase-space mismatch. The beam is constantly excited by the phase-space mismatch when the tunes are equal. The phase-space mismatch induces emittance dilution in both the horizontal and vertical planes.

\section{Effect of dipole field errors}

The effect of dipole field error produces coherent beam oscillation. Nonlinearities in the accelerator converts the coherent beam oscillation into emittance dilution. Figure 35 shows the effect of emittance evolution in the presence of space-charge force with and without dipole field errors. Since the horizontal dipole field error can be produced by dipole roll, we expected to have a larger $a_{0}$ term than the corresponding $b_{0}$ term. We estimated that the

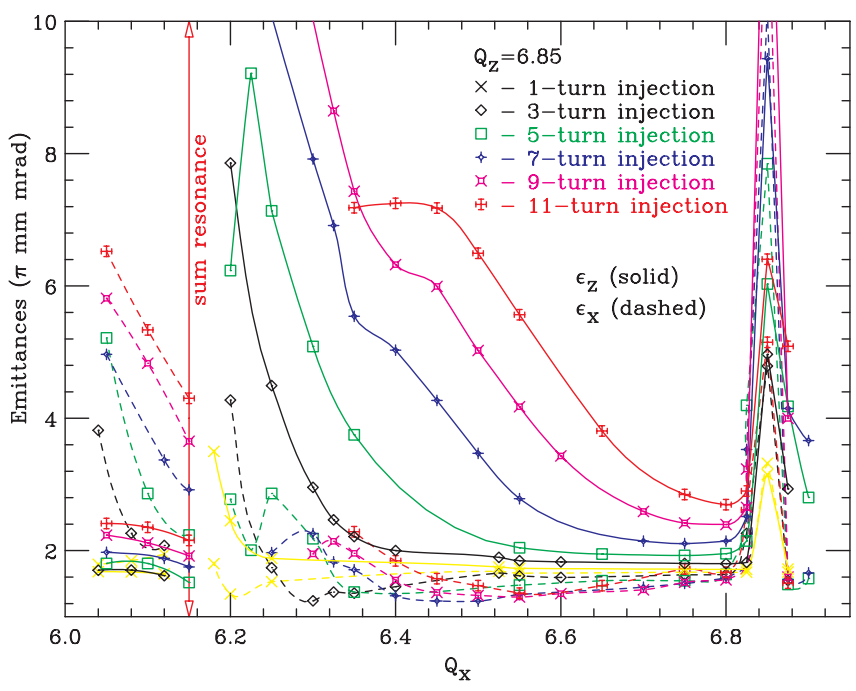

FIG. 34. (Color) The horizontal (dashed line) and vertical (solid line) emittances are plotted as a function of the horizontal tune for different beam intensities (from 1-turn to 11-turn injections). 


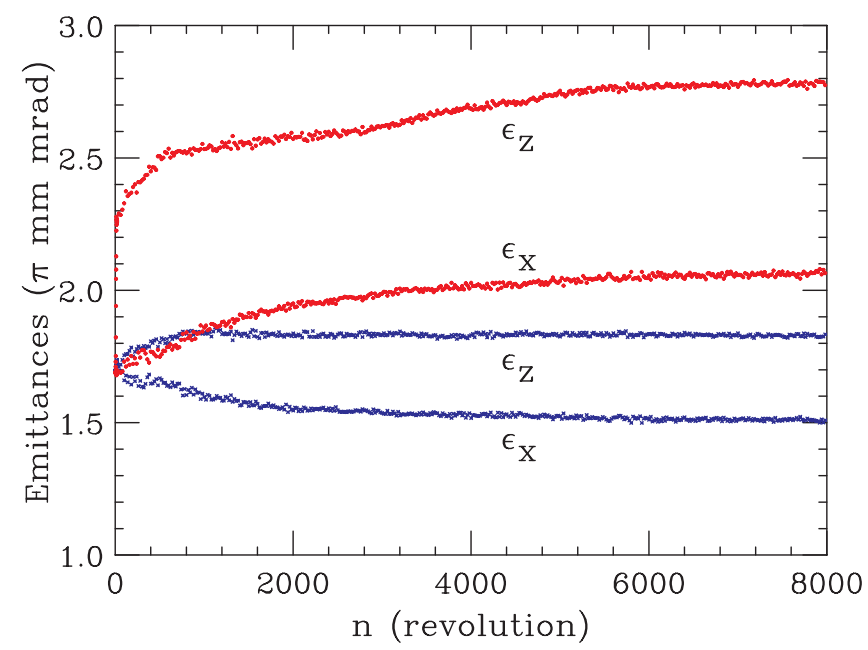

FIG. 35. (Color) The emittance evolution for a beam with 12turn injection for the case with random dipole field error (red), and without the dipole field error (blue). The random dipole field error has an amplitude of $2.0 \times 10^{-5} \mathrm{rad}$ and $7.5 \times 10^{-5} \mathrm{rad}$ for the horizontal and vertical planes, respectively.

amplitudes of dipole error are about $2.0 \times 10^{-5} \mathrm{rad}$ for horizontal motion and $7.5 \times 10^{-5}$ rad for vertical motion, respectively. A large $a_{0}$ term produces a larger vertical beam emittance increase as shown in Fig. 35. Had we have a large $b_{0}$ term, we would have produced a large horizontal emittance increase.

\section{Effect of rf cavities in the dispersive region}

It is known that the quantum fluctuation induced by synchrotron radiation loss in dipole is the main source of excitation for electron beams in storage rings. The acceleration of localized rf cavities in the Fermilab Booster causes coherent beam betatron oscillation about the closed orbit as shown in Fig. 26. However, the effective kick angles of these cavities are less than $1 \times 10^{-5} \mathrm{rad}$, its effect is small. If we had all cavities located in a single straight section, the resulting increase of emittance would be about $0.5 \pi \mathrm{mm}$ mrad during the acceleration. Since these cavities are distributed in 10 straight sections, its effect on emittance is small.

\section{Putting it all together}

When the space-charge force is included during the injection process, the phase-space ellipse of the particle motion becomes mismatched. The magnitude of mismatch is proportional to the space-charge perveance $K_{\mathrm{sc}}$. The phase-space mismatch of each particle will decohere and the resulting beam distribution will reach a larger emittance. Thus the emittance growth rate is proportional to the space-charge perveance, i.e., $d \epsilon / d t \sim K_{\mathrm{sc}}$, where $t$ is the revolution number. This may explain the reason why we were able to fit the vertical beam emittance data with the model. Figure 36 shows the rms beam radii at a focusing

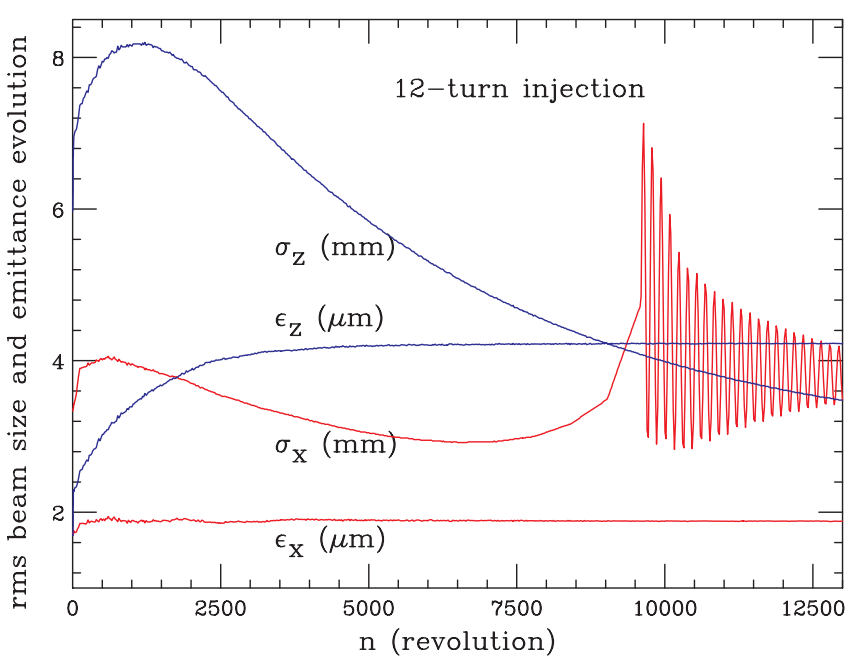

FIG. 36. (Color) The emittance evolution for a beam with 12turn injection when the space-charge force is introduced.

quadrupole location and the emittance evolution for the beam intensity at 12-turn injection. The parameters used in the numerical simulations are $2.0 \times 10^{-5} \mathrm{rad}$ and $7.5 \times$ $10^{-5}$ rad dipole error for the horizontal and vertical motion, respectively, $4.0 \times 10^{-4} \mathrm{~m}^{-1}$ quadrupole field error, and $35 \times 10^{-4} \mathrm{~m}^{-1}$ for the skew-quadrupole field error. The characteristic of the vertical emittance growth clearly resembles that of the experimental data shown in Fig. 10.

The emittance dilution occurs mainly in the vertical plane. This has resulted from the combination of the random skew quadrupoles, Montague resonance and a larger dipole error for the vertical motion (than that for the horizontal motion). The situation can be different if the machine operation condition is changed. The Fermilab Booster has been optimized by many years of operational experiences. The current mode is certainly an acceptable one. The contribution of emittance growth for the 12-turn injection is $50 \%$ due to the skew quadrupole, $25 \%$ dipole field error, and $25 \%$ from the intrinsic space-charge effects (Montague resonance). The effect of large skewquadrupole contribution is due to a large space-charge tune shift, and a large linear sum resonance at $\nu_{x}+\nu_{z}=$ integer resonance as shown in Fig. 32.

We now compare the emittance evolution for various beam intensities. Figure 37 compares the evolution of the vertical emittances for 6, 12, and 16-turn injections. Note that the emittance growth resembles that shown in Fig. 10.

\section{Other observations}

We have found that the horizontal rms bunch width oscillates with twice the synchrotron frequency due to mismatch in the rf potential well after the transition-energy crossing. We found that this mismatched horizontal bunchwidth oscillation could actually occur before the transition energy if the beam intensity is high. 


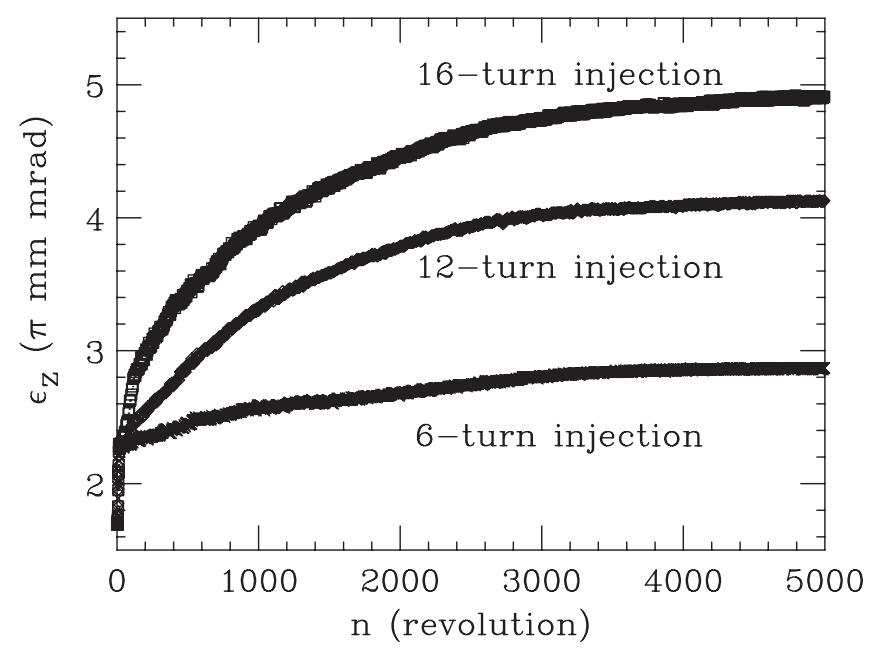

FIG. 37. The evolution of the vertical emittance for beams with 6,12 , and 16-turn injections. The emittance evolution can be compared with those of Fig. 10.

The vertical beam width showed also post-transition oscillation (see Fig. 6). The post-transition vertical beam width oscillation is out of phase with that of the horizontal plane. This may have resulted from the Montague resonance. However, using the betatron tunes measured from independent component analysis method, we do not observe the vertical emittance oscillation in our numerical simulation, unless the betatron tunes are nearer to each other at the transition-energy crossing.

\section{CONCLUSION}

We have carried out systematic IPM measurements at the Fermilab Booster for the first time to understand the emittance evolution for space-charge-dominated beams. The vertical emittance has a very strong dependence on the beam intensity with $d \epsilon_{z} / d t \sim b_{1}+K_{\mathrm{sc}}$, where $K_{\mathrm{sc}}$ is the space-charge perveance. Using the scaling laws of the betatron emittance and off-momentum spread, we have also succeeded in separating the horizontal emittance and the rms off-momentum spread from the horizontal beam width. We found that the horizontal emittance is less dependent on the space charge.

We use the rms space-charge potential model to carry out systematic numerical simulation to understand the observed phenomena. We found that the vertical emittance growth is induced mainly by random skew quadrupoles in the presence of a large space-charge tune spread. The sum and difference linear-coupling resonances play a very important role in the emittance growth for a fast-ramping accelerator. The Montague resonance plays a minor role in the vertical emittance growth. The random dipole field error for the vertical plane, due mainly to the dipole roll, has contributed a role in the vertical emittance growth. The random quadrupole error is not important at the Fermilab
Booster because the betatron tunes are far away from the stop bands [21].

Although we can use our numerical-simulation model to understand the main source of emittance growth at the Fermilab Booster, we do not have the explanation of the slow growth of the emittance at about $1 \pi \mathrm{mm} \mathrm{mrad}$ in $10^{4}$ revolutions. One could introduce an rf-noise model in dipole or quadrupole fields to produce this emittance growth. Since we did not know the source, we decided not to put it in our model.

As we found that the sum and difference linear-coupling resonances induced by the skew quadrupoles are the major source of emittance growth at the Fermilab Booster, it would be very important to carry out additional emittance measurements with skew-quadrupole correction scheme, which minimizes the sum and difference stop bands. We think that the beam loss at the Fermilab Booster arises essentially in the longitudinal phase space, and that the skew-quadrupole correction scheme may be able to reduce the vertical emittance growth, but may not be able to minimize the beam loss. To minimize beam losses, one needs to chop the linac beam pulse as the source and employ longitudinal phase-space painting in the longitudinal phase space at injection. Work on experiment with skew-quadrupole correction schemes will be carried out in the near future.

\section{ACKNOWLEDGMENTS}

This work is supported in part by grants from the U.S. Department of Energy, under Contracts No. DE-AC0276CH03000 and No. DE-FG0292ER40747 and the National Science Foundation No. NSF PHY-0244793. One of the authors (S.Y.L.) thanks supports from the Humboldt Foundation in Germany and the Indiana University, USA. He also thanks Professor I. Hofmann for helpful discussions and GSI for kind hospitality during this research leave.

[1] F. J. Sacherer, Ph.D. thesis, U.C. Berkeley [Report No. UCRL-18454, 1968].

[2] S. Y. Lee, Accelerator Physics (World Scientific, Singapore, 2004), 2nd ed.

[3] High Intensity and High Brightness Hadron Beams, edited by I. Hofmann, J. M. Lagniel, and R. W. Hasse, AIP Conf. Proc. No. 773 (AIP, New York, 2004).

[4] Space-Charge Dominated Beams and Applications of High Brightness Beams, AIP Conf. Proc. No. 377, edited by S. Y. Lee (AIP, New York, 1996).

[5] B. W. Montague, CERN Report No. 68-38, CERN, 1968; I. Hofmann et al., in Ref. [3], p. 169; I. Hofmann et al.., in Proceedings of the Particle Accelerator Conference, Knoxville, TN, 2005 (IEEE, Piscataway, NJ, 2005), p. 330.

[6] S. Cousineau, Ph.D. thesis, Indiana University, 2002; S. Cousineau, et al., Phys. Rev. ST Accel. Beams, 6, 034205 (2003). 
[7] X. Huang, S. Y. Lee, Eric Prebys, and Chuck Ankenbrandt, in Proceedings of the Particle Accelerator Conference, Knoxville, TN, 2005 (Ref. [5]).

[8] X. Huang, S. Y. Lee, E. Prebys, and R. Tomlin, Phys. Rev. ST Accel. Beams 8, 064001 (2005).

[9] X. Huang and S.Y. Lee, Fermilab Technical Memo No. FERMILAB-TM-2326-AD, 2005.

[10] J. Amundsen et al., Phys. Rev. ST Accel. Beams 6, 102801 (2003). The computer code from James Amundson was used to fit the profile data and calibrate the beam width.

[11] See, e.g., A. Piwinski, CERN Report No. CERN-92-01, 1992, p. 226.

[12] M. Reiser, Theory and Design of Charged Particle Beams (Wiley, New York, 1994), see Eq. (6.186).

[13] S. Ohnuma, Fermilab Technical Memo No. TM-1381-A, 1986.
[14] S. Y. Lee and J. M. Wang, in Proceedings of the Particle Accelerator Conference, Vancouver, Canada (IEEE, Piscataway, NJ, 1985), p. 2323.

[15] D. Huang, S. Y. Lee, and K. Y. Ng, Fermilab Report No. Fermilab-FN-0774-AD, 2005.

[16] A. Sorrenssen, Part. Accel. 6, 141 (1975).

[17] W. Guo and S. Y. Lee, Phys. Rev. E 65, 066505 (2002); M. H. Wang, Y. Sato, and S. Y. Lee, in Proceedings of the Particle Accelerator Conference, Portland, OR, 2003 (IEEE, Piscataway, NJ, 2003), p. 3240.

[18] M.H. Wang, and S. Y. Lee, J. Appl. Phys. 92, 555 (2002).

[19] Bill Pellico (private communication).

[20] S. Kheifeit, PETRA Note No. 119, 1976.

[21] X. Huang and S. Y. Lee, Fermilab Technical Memo No. FERMILAB-TM-2327-AD, 2005. 